\title{
Hybrid Cyclicity: Combining The Benefits Of Cyclic And Non-Cyclic Timetables
}

\author{
Tomáš Robenek * Shadi Sharif Azadeh * Yousef Maknoon * \\ Michel Bierlaire *
}

May 10, 2016

Report TRANSP-OR 160510

Transport and Mobility Laboratory

École Polytechnique Fédérale de Lausanne

transp-or.epfl.ch

*Transport and Mobility Laboratory (TRANSP-OR), School of Architecture, Civil and Environmental Engineering (ENAC), École Polytechnique Fédérale de Lausanne (EPFL), CH-1015 Lausanne, Switzerland, \{tomas.robenek, shadi.sharifazadeh, yousef.maknoon, michel.bierlaire\} @epfl.ch 


\begin{abstract}
We propose a new type of timetable that would combine both the regularity of the cyclic timetables and the flexibility of the non-cyclic ones. In order to do so, several combinations of the two timetables are considered. The regularity is incorporated in their design and the flexibility is evaluated using the passenger satisfaction (in monetary units). Each of the tested timetables is constructed using the Passenger Centric Train Timetabling Problem, that is solved using a simulated annealing heuristic. The performance of each of the considered timetables is assessed on the real network of Israeli Railways. The results of the case study show that our proposed hybrid cyclic timetable can provide the benefits of the cyclic and the non-cyclic timetable simultaneously. This timetable consists of $75 \%$ of cyclic trains (securing the regularity of the service) and of $25 \%$ of non-cyclic trains (deployed as supplementary trains during the peak hours and capturing the demand fluctuation). The level of the passenger satisfaction of the hybrid cyclic timetable is similar to the level of the non-cyclic one, which has about $18.5 \%$ of improvement as compared to the purely cyclic one.
\end{abstract}

Keywords: Railway Timetable, Cyclicity, Passenger Satisfaction, Simulated Annealing, Passenger Centric Train Timetabling Problem

\title{
Acknowledgement
}

We would like to thank Mor Kaspi and Tal Raviv for providing us with the demand data of the Israeli network and for their kind explanations of the aforementioned data. 


\section{Introduction}

A train timetable is the main product of a Train Operating Company (TOC). It consists of the departure times of every train from each of its stopping stations. When creating a timetable the TOCs usually focus on its operational aspects rather than the actual passenger demand that it is supposed to serve. According to the literature, two types of timetables (in passenger railway service) exist: cyclic and non-cyclic.

The cyclicity originates from the Periodic Event Scheduling Problem (PESP), where a given set of events is scheduled in equally spaced intervals (first defined in Serafini and Ukovich (1989)). In the case of passenger railway service, a special type of cyclic timetables is the clock-faced timetable, where the cycle is one hour. It is especially popular within railways, following the assumption that such timetables are easy to remember and thus preferred by the passengers. Some studies confirm that indeed a regularity of a timetable leads to an increase in the passenger demand (Wardman et al. (2004), Johnson et al. (2006)). However, such a timetable design provides an inefficient operation system as there is a mismatch between the supply (determined by the timetables) and the demand (characterized by the time dependent passengers arrival rate).

In the non-cyclic timetable, on the other hand, no special rule is imposed on the departure time of the trains (see Caprara et al. (2002) for instance). This makes the non-cyclic timetable more flexible in accounting for the passenger demand. A recent study of Robenek et al. (2016) shows that this is especially true for high volumes of passengers. The flexibility has been compared based on the passenger satisfaction. But given the structure of a non-cyclic timetable (no repeating pattern), it might discourage some passengers from choosing the train as their mode of transport.

Given the above, one cannot make a statement about the superiority of one timetable over the other. A combination of the two is needed. In this paper, we investigate hybrid timetables that combine the benefits of both - the regularity of the cyclic timetable and the flexibility of the non-cyclic one. The approach consists in imposing various levels of cyclicity and to evaluate the corresponding flexibility.

The timetables are obtained by solving the Passenger Centric Train Timetabling Problem (PCTTP) using a large neighborhood search heuristic combined with simulated annealing. The performance of each of the newly proposed timetables is assessed and compared to the cyclic and non-cyclic timetables on the real network of Israeli Railways.

The manuscript is structured as follows: in the literature review (Section 2), a survey on train timetabling models is presented. The PCTTP model to construct a timetable while maximizing the passenger satisfaction is introduced in Section 3. The definitions of the existing types of the timetables and the constraints that they impose on the PCTTP model are given in Section 4. Similarly in Section 5, the hybrid timetables and their impacts on the PCTTP are discussed. Section 6 provides the insights about the solution methodology that is used to obtain the results for our case study in Section 7. The paper is finalized by drawing some conclusions and discussions of possible extensions in Section 8.

\section{Literature Review}

Since the goal of this study is a new type of timetable, the literature review focuses on the timetable design, which is typically done by solving the Train Timetabling Problem (TTP). The main goal of 
the TTP is to resolve any potential track occupation conflicts among the trains, in order to construct an operational timetable. Two versions of this problem exist in the literature: cyclic and non-cyclic. The difference between the two versions is that the cyclic TTP imposes additional rules on the departure times.

\subsection{Non-Cyclic TTP}

This version of the problem is either formulated as an Integer Linear Programming (ILP) or as a Mixed Integer Linear Programming (MILP). The ILP model uses discretized time, whereas the MILP model uses continuous time.

Model One of the main differences, between the two formulations, is the way how they handle the conflicts among the trains. The ILP model discretizes the time and splits the lines into blocks (Brannlund et al. (1998)). One train at a time can occupy a block. Clique constraints are then imposed to secure the safety (Caprara et al. (2002, 2006), etc.). Their number grow exponentially with the size of the problem. Several other ILP re-formulations exist (Cacchiani et al. (2010a)). The ILP formulation can also allow for the scheduling of extra freight trains within the planning of the passenger service (Cacchiani et al. (2010b)).

The MILP model, on the other hand, is considering the departure times as continuous variables. The minimum headway between two trains is secured using binary variables that indicate the order of the trains in which they leave from a given station (Carey and Lockwood (1995), Higgins et al. (1997), etc.).

Objective Function For the ILP formulations, it is assumed that ideal timetables are known a priori. The ideal timetable is defined as the most profitable one. The objective of the problem is to minimize the changes made to the ideal timetables (Caprara et al. (2002, 2006, 2007), etc.). Note that we did not find in the literature a methodology to create such an ideal timetable.

The MILP formulations have various objective functions: minimization of the overall cost associated with the allocation of a train path (Carey and Lockwood (1995)), minimization of the total weighted travel time (Higgins et al. (1997)), minimization of the delays on the arrival and departure times (Harrod (2012)), mimimization of the deviation from the originally planned timetable expressed as a delay (Oliveira and Smith (2000)), minimization of the feasibility violation by penalization (Burdett and Kozan (2010)), etc.

A recent approach consists in relaxing the assumptions that the passengers would always take their shortest path and thus use the total travel time minimization as the objective (Schmidt and Schöbel (2015)). This approach is further extended by taking into account the whole passenger satisfaction, which is to be maximized (Robenek et al. (2016)).

Solution Approach A common methodology to solve the ILP formulation is the lagrangian relaxation of the track capacity constraints (Brannlund et al. (1998); Caprara et al. (2002, 2006); Cacchiani 
et al. (2012)). However, the column generation framework seems to find better bounds than the aforementioned relaxation (Cacchiani et al. (2008)). One may also use dynamic programming to solve the clique constraints (Cacchiani et al. (2013)).

For the MILP formulation, the literature agrees on using a heuristic as a common solution approach: a heuristic that considers one train at a time to solve the MILP based on the already scheduled trains (Carey and Lockwood (1995)), as well as local search, tabu search, genetic and hybrid heuristics (all presented in Higgins et al. (1997)), among others.

\subsection{Cyclic TTP}

The aim of the cyclic TTP is to resolve any potential track occupation conflicts and to make the departure times of all trains cyclic, i.e. the departure time of every train on a given line is equally spaced with an interval of a cycle. This class of problems is referred to as Periodic Event Scheduling Problems (PESP). The PESP were first defined by Serafini and Ukovich (1989). Due to the nature of the problem (repeating pattern), it is sufficient to solve the cyclic TTP for one cycle only (typically the peak hour as it is the most dense part of the day) and repeat the solution in every other cycle. A special case of a cyclic timetable is the clock-faced timetable, where the cycle is equal to one hour (typical for railways).

Model The majority of the cyclic TTP formulations are based on the PESP. In some cases, the PESP is extended with additional constraints such as train synchronization (Peeters (2003)) or symmetry (Liebchen (2004)). The symmetry can be described as follows: two trains of the same line traveling in opposite directions meet at time 0 , i.e. if a train leaves at 14th minute of the cycle in one direction, then the train in the opposite direction leaves from the same station at time 46 for a cycle of one hour. The sum of the departure times at any station is equal to the cycle. The symmetry is a popular measure in Switzerland and Germany.

The basic PESP formulation considers one universal cycle, known a priori, for the whole railway network. However, one may consider to allow for varying sizes of the cycle over the planning horizon (Zhong et al. (2013)) or to make the size of the cycle a decision variable (Heydar et al. (2013)). The proposed measures have the intention of securing better transfers or to maximize the utilization of the infrastructure respectively.

Objective Function While the model of the cyclic TTP is well defined and recognized, the same can not be said about the objective function of the problem. In the very beginning, there were models without any objective (Odijk (1996)), thus an ad-hoc feasible solution would be generated. Later on, an objective function that would minimize the total waiting time has been introduced (Nachtigall (1996); Nachtigall and Voget (1996); Yang et al. (2010), etc.). In some studies, the waiting time has been further decomposed among the different types of passengers such as transferring, throughput and others (Vansteenwegen and Oudheusden (2006, 2007)). The focus on the waiting time only came from the assumption that the passengers would always take their shortest path between their origin and destination, thus rendering the total travel time irrelevant. In the recent literature, this assumption has been released and the cyclic TTP integrates the routing of the passengers. One can either minimize the 
total travel time of the passengers (Hoppmann et al. (2015)), to maximize the number of transported passengers (Cordone and Redaelli (2011)) or to maximize the passenger satisfaction (Robenek et al. (2016)).

Solution Approach Similarly to the objective function, one superior methodology for solving the problem does not exist. The applied techniques span from the semi/exact methods, such as branch and bound (Nachtigall (1996)), constraint generation (Odijk (1996)), modulo simplex (Nachtigall and Opitz (2008)), cycle periodicity reformulation (Peeters (2003); Liebchen and Peeters (2009)), satisfiability reformulation (Großmann et al. (2012)), etc., to heuristic methods, such as genetic algorithm (Nachtigall and Voget (1996)), and simulated annealing with particle swarm optimisation (Jamili et al. (2012)).

Remark Due to the restrictive (cyclicity) constraints, a feasible solution might not always be obtained. Two options for correction exist: the adjustment of the underlying assumptions (to keep the cyclicity intact) or allowing for some degrees of irregularity in the timetable. One of the assumptions that can be changed is the running time of a train in between two stations. Typically, the cyclic TTP treats it as a fixed input, but it can be turned in to a decision on an interval of a minimal and a maximal value and possibly result into having a feasible solution (Kroon and Peeters (2003)). The other option is to affect the cyclicity itself. When a railway network is highly dense (such as in China), one can decide to have some lines cyclic and the others non-cyclic. This framework has been proposed by Yang et al. (2010), where the cyclicity is primarily given to the most busy lines in terms of the transported passengers. This approach would make a whole train line non-cyclic, but sometimes it might be sufficient to allow only for a small deviation from the cyclic departure time of only a few trains. Such a framework is proposed by Caimi et al. (2011), where the problem has a periodic service as an intention and not as a hard constraint.

\subsection{Summary}

From the above survey, we have learned that most often the goal is to provide either a fully non-cyclic or a fully cyclic timetable. A partially cyclic timetable is considered, only when no feasible solution can be obtained. However, a new direction of relaxing the cyclicity to achieve the benefits of the non-cyclic timetables is emerging. In this paper, we are going to further exploit this concept.

\section{Timetable Construction}

A timetable, in the passenger railway service, is formally defined as a set of arrival and departure times of every train at each of its stopping stations. The frequency (number of trains) and the stopping patterns are given by the Line Planning Problem (LPP, Schöbel (2012)) and are fixed. By imposing rules on these times, one can obtain different types of timetables. At first, we present a timetable design approach (irrespective of the timetable type) which is based on the overall passenger satisfaction. The existing types of timetables are presented in Section 4 and our proposed types of timetables are 
presented in Section 5. For the convenience of the reader, we organize all mathematical notation by its type and order of appearance in Table 1 at the end of this section.

The passenger satisfaction serves as the indicator of the flexibility of a timetable. In order to construct a timetable, we use the model of the Passenger Centric Train Timetabling Problem (PCTTP) proposed by Robenek et al. (2016). In PCTTP, the objective is to construct a timetable (either cyclic or non-cyclic) that would maximize the overall passenger satisfaction. The model combines the timetable design with the routing of the passengers. Unlike the traditional TTP, this model does not deal with the conflicts among trains. The time is discretized into minutes.

The inputs of the PCTTP can be categorized into two groups: the network layout and the passenger demand data. The network layout consists in the train lines given by the LPP. A line $\ell \in \mathrm{L}$ is an ordered set of stopping stations, that includes the dwell times at the stations and the travel times in between. Each line has an assigned frequency by the LPP, i.e. the set of available trains $v \in \mathrm{V}^{\ell}$ serving the line $\ell$ during a given time horizon $h$ in minutes, which remains fixed. From now on, we refer to a train as a combination of indices $(\ell, v)$. Based on the lines, the set of paths $p \in P_{i}$ for all Origin-Destination (OD) pairs $i \in \mathrm{I}$ is given. An example of an algorithm to construct the set of paths is presented in Appendix A. A path consists in an ordered set of lines $\mathrm{L}^{p}$ to be traversed from an origin to a destination with a given train of each line $(\ell, v)$. Different trains on the same line constitute different paths. Each line can be further decomposed into unique segments. A segment is the part of the infrastructure, where a train does not stop. A train $(\ell, v)$ is characterized by its line $\ell$, departure time $d_{v}^{\ell}$ from the origin of the line in minutes (the travel times and dwell times are fixed) and its capacity that cannot be exceeded on any of the traversed segments. Unlike the TTP, the PCTTP model considers the dwell times fixed, hence a decision on the departure time from the origin station of each line is sufficient.

As for the passengers, they can form groups that share the same OD pair $i \in I$ and preferred arrival time to the destination $t \in T_{i}$. A passenger group is denoted as $(i, t)$ and contains $n_{i}^{t}$ passengers. The model does not allow splitting of the groups. However, one may create groups of size one, in order to treat the passengers individually. The actual value of the preferred arrival time to the destination is given by the parameter $a_{i}^{t}$. The routing of each passenger group is determined based on the available paths $p \in P_{i}$ from the origin to the destination. Within each path, a passenger group is given a minimum transfer time $m$, in order to be able to realize a transfer from one train to another in the transferring stations. Any other additional time spent in the transferring stations is counted as the waiting time $w_{i}^{\text {tp }}$. A passenger group has to use exactly one path to get from an origin to a destination. When no path is available within the planning time horizon $h$, due to the train capacity issues, a socalled penalty path $\mathcal{P}_{i}^{t}$ is offered. The penalty path consists in the shortest path between the given OD pair realized after the end of the planning horizon $h$.

The satisfaction of a passenger group $(i, t)$ using a path $p$ is denoted as $\mathcal{S}_{i}^{\text {tp }}$. The value of $\mathcal{S}_{i}^{\text {tp }}$ depends on four attributes considered by the passengers: the in-vehicle-time, the waiting time at transfers, the number of transfers and the schedule passenger delay. The in-vehicle-time is the sum of the running times $r_{i}^{p \ell}$ of all train lines $\ell$ used in the path $p$, when traveling between OD pair $i$. Note that the running times have the same value for different trains of the same line, but differ among the OD pairs. The different OD pairs get on and off the train line at different stations. Similarly for the paths, different paths within the same OD pair use either different lines or use the same lines, but transfer from one to another in different stations. The waiting time $w_{i}^{\text {tp }}$ is the sum of all waiting times 
within the path. The number of transfers is equal to the number of train lines in the path minus one $\left(\left|\mathrm{L}^{\mathrm{p}}\right|-1\right)$. The schedule passenger delay indicates whether the passenger group has arrived early to the destination $\left(\max \left(a_{i}^{t}-\delta_{i p}^{t}, 0\right)\right)$, on time $(=0)$ or late $\left(\max \left(0, \gamma_{i p}^{t}-a_{i}^{t}\right)\right)$. Note that the schedule passenger delay is related to the preferred arrival time to the destination and that it is not a delay of the train.

Each of the above attributes is transformed into in-vehicle-time units using parameters collected from the literature $\left(\beta_{\mathrm{W}}, \beta_{\mathrm{T}}, \beta_{\mathrm{E}}\right.$ and $\left.\beta_{\mathrm{L}}\right)$. Details are provided in Appendix A. The sum of these transformed attributes constitutes the generalized time (in minutes), that can be transformed into the generalized cost (in monetary units) by multiplying it with the Value-Of-Time (VOT). The VOT is an indicator of how many of monetary units are the passengers willing to pay, in order to achieve given savings in time. For instance in Israel, the commuting passengers are willing to pay $21.12 \mathrm{New}$ Israeli Shekels (estimated in 2012 by Shiftan et al. (2008)) for a saving of one hour. The lower the generalized cost is, the higher is the passenger satisfaction. Based on the above description, the passenger satisfaction of a path $p$ with respect to the passenger group $(i, t)$ is formulated as follows:

$\mathcal{S}_{i}^{\mathrm{tp}}=-\operatorname{VOT} \cdot\left(\sum_{\ell \in \mathrm{L}^{\mathrm{p}}} \mathrm{r}_{\mathrm{i}}^{\mathrm{p} \ell}+\beta_{\mathrm{W}} \cdot w_{\mathrm{i}}^{\mathrm{tp}}+\beta_{\mathrm{T}} \cdot\left(\left|\mathrm{L}^{\mathrm{p}}\right|-1\right)+\beta_{\mathrm{E}} \cdot \delta_{\mathfrak{i p}}^{\mathrm{t}}+\beta_{\mathrm{L}} \cdot \gamma_{\mathrm{ip}}^{\mathrm{t}}\right), \quad \forall \mathrm{i} \in \mathrm{I}, \forall \mathrm{t} \in \mathrm{T}_{\mathrm{i}}, \forall \mathrm{p} \in \mathrm{P}_{\mathrm{i}}$,

By solving the PCTTP model, one obtains the exact timetable as well as the subsequent routings of the passengers through the network. The decision of which passenger group is assigned to which path is carried out by the model. This decision is driven by the objective function (passenger satisfaction maximization). The result is a system optimum. The PCTTP as such generates a non-cyclic timetable. It is needed to include additional cyclicity constraints (described in the next section) to obtain a cyclic timetable. Since the PCTTP provides the routing of the passengers, the profit of a train operating company associated with the resulting timetable can be estimated. However, given that it is assumed that the passengers have railways as their only mode of transport (the penalty path consists in trains), the demand is inelastic. Thus the profit for different timetables is similar. For this reason, we omit the profit estimation in this study.

\begin{tabular}{|c|c|c|c|}
\hline Name & Description & Units & Type \\
\hline L & set of operated train lines given by the LPP & - & set \\
\hline $\mathrm{V}^{\ell}$ & set of available trains for the line $\ell$ (frequency) & - & set \\
\hline I & set of origin-destination pairs & - & set \\
\hline$P_{i}$ & set of possible paths between OD pair $i$ & - & set \\
\hline $\mathrm{L}^{\mathrm{p}}$ & set of lines in the path $p$ & - & set \\
\hline $\mathrm{T}_{\mathrm{i}}$ & set of preferred arrival times for OD pair $i$ & - & set \\
\hline$(\ell, v)$ & a train $v$ serving the line $\ell$ & - & index \\
\hline$(i, t)$ & $\begin{array}{l}\text { a passenger group traveling between OD pair } i \text { with a pre- } \\
\text { ferred arrival time } t\end{array}$ & - & index \\
\hline $\mathrm{K}$ & set of cycles within the planning horizon & - & set \\
\hline $\mathrm{h}$ & duration of the planning horizon & $\min$ & parameter \\
\hline
\end{tabular}




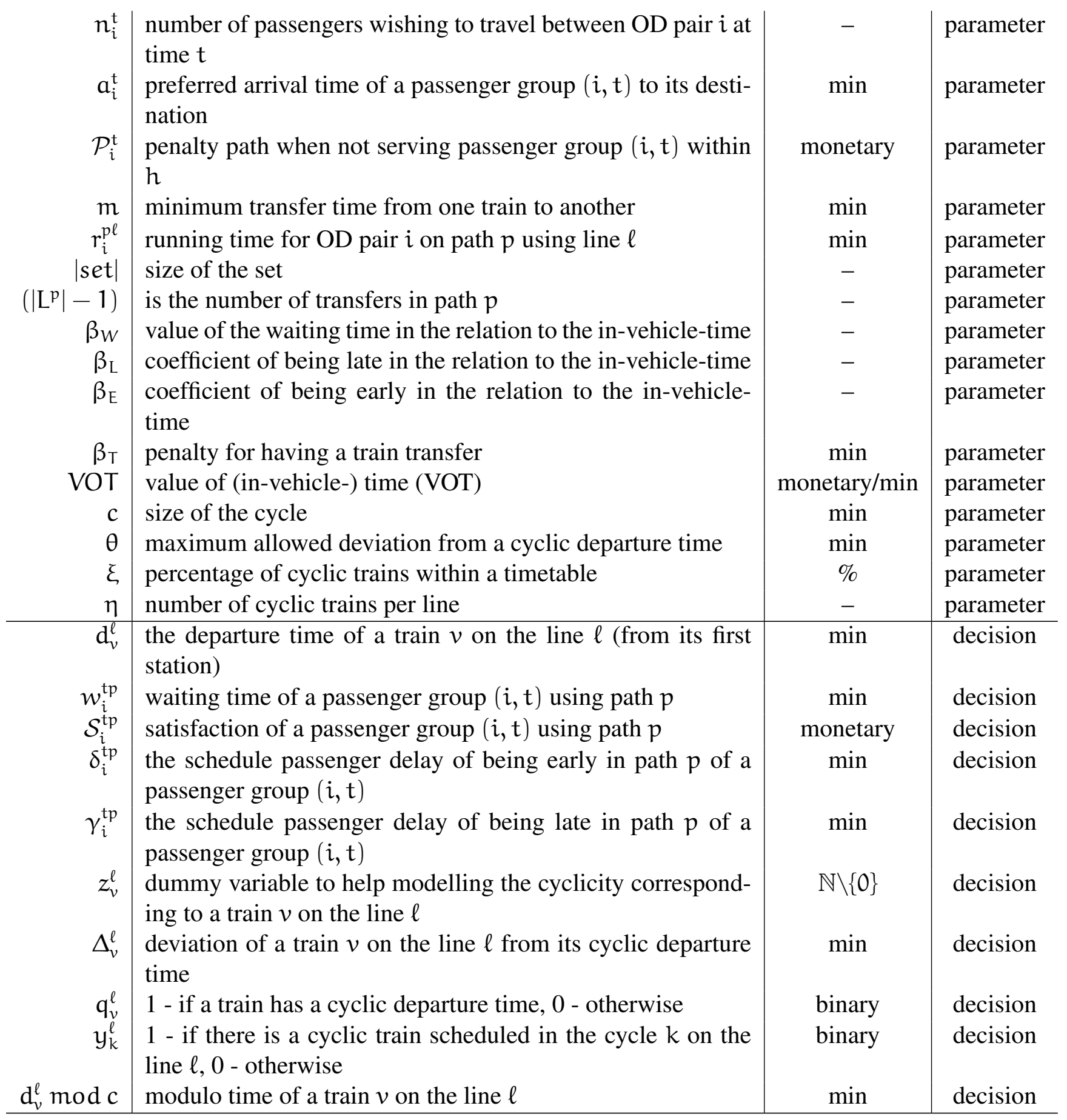

Table 1: Mathematical notations 


\section{Existing Timetables}

As mentioned in the previous section, we define a timetable as a set of departure times $\mathrm{d}_{v}^{\ell}$ of every train $v \in \mathrm{V}^{\ell}$ from its origin station on every line $\ell \in \mathrm{L}$. Even though the lines consist of several stopping stations, in the PCTTP it is sufficient to decide only the departure time of every train from the origin station of a line, as the arrival times to the subsequent stations are derived from the fixed travel times and dwell times. By imposing rules on train departure times, one can obtain two types of timetables: cyclic and non-cyclic.

Cyclic Timetable The cycle $\mathrm{c}$ represents the difference between the departure times of two consecutive trains on every line. It is given in minutes and its value holds for the whole timetable. When varying size of the cycle is needed, one can either solve a TTP model of variable cycles (Zhong et al. (2013)) or (more often) to conceptually split a line into several ones. However, the split of a line is viable only when the various sizes of the cycle are its multiples. In railways, the typical value of the cycle $c$ is 60 minutes. The regularity is enforced by including the cyclicity constraints in the model. They can take the following form:

$$
\mathrm{d}_{v}^{\ell}-\mathrm{d}_{v-1}^{\ell}=\mathrm{c}, \quad \forall \ell \in \mathrm{L}, \forall v \in \mathrm{V}^{\ell}: v>1 .
$$

Adding cyclicity constraints to the model creates a strictly cyclic timetable, i.e. once the operation of a line $\ell$ has started, there would be a train departing in every cycle. An example of such a timetable is shown in Table 8 (in Appendix B). This type of timetable is not always desirable. The passenger demand is time dependent and is lower in between the peak hours. Therefore operators might choose to interrupt their operation for the duration of the off-peak hours. In this case, constraints (2) can be modified to a version where the departures of two consecutive trains are spaced in multiples of cycles $z_{v}^{\ell}$. In other words, the difference between two consecutive trains can now have values of 60, 120, 180, etc. minutes. The values of these variables are integers with the lowest value being 1 . The constraints (2) are reformulated as:

$$
\mathrm{d}_{v}^{\ell}-\mathrm{d}_{v-1}^{\ell}=\mathrm{c} \cdot z_{v}^{\ell}, \quad \forall \ell \in \mathrm{L}, \forall v \in \mathrm{V}^{\ell}: v>1 .
$$

In this manuscript, we name a PCTTP containing constraints (3) as cyclic. An example of such a timetable is presented in Table 7 (in Appendix B).

Non-Cyclic Timetable In the non-cyclic timetable, no rule is enforced on the departure times of trains. However, one can add the below constraints, in order to avoid the symmetry in the model.

$$
\mathrm{d}_{v}^{\ell} \leq \mathrm{d}_{v+1}^{\ell}-1, \quad \forall \ell \in \mathrm{L}, \forall v \in \mathrm{V}^{\ell}: v<\left|\mathrm{V}^{\ell}\right| .
$$

No other buffer time between two consecutive trains, than the above one minute, is needed. The decision, if two trains are being scheduled close to each other, is driven by the objective function. Note, that the PCTTP model does not handle potential conflicts among trains. Since no rule on the 
departure times is enforced, the non-cyclic timetables are better in accordance with the time dependency of the demand. Therefore, the non-cyclic timetable is more flexible than the cyclic one. An example of such timetable is shown in Table 10 (in Appendix B).

\section{Hybrid Timetables}

In this section, we introduce the concept of hybrid timetables which are a combination of cyclic and non-cyclic timetables. Each type of hybrid timetable is described in the form of the additional constraints that are imposed on the original PCTTP formulation. The regularity of the hybrid timetables is taken care of by design and the flexibility is evaluated using the passenger satisfaction upon solving the respective PCTTP formulation. Later on (in Section 7), we test and evaluate these timetables against each other (including the existing ones) using real case data.

$\theta$ Shifted Cyclic Timetable The first hybrid timetable is inspired by Caimi et al. (2011). They allow small deviations from the cyclic departure times, in order to obtain a feasible solution. We introduce a variable $\Delta_{v}^{\ell}$ capturing the deviation from the cyclic departure time of a train $v$ on line $\ell$ given in minutes. This deviation is restricted within the bounds $[-\theta, \theta]$ in minutes, in order to control it. To obtain such a timetable for a given $\theta$, we add constraints (5) and (6) in the model of the PCTTP.

$$
\begin{array}{cl}
\left(\mathrm{d}_{v}^{\ell}-\Delta_{v}^{\ell}\right)-\left(\mathrm{d}_{v-1}^{\ell}-\Delta_{v-1}^{\ell}\right)=\mathrm{c} \cdot z_{v}^{\ell}, & \forall \ell \in \mathrm{L}, \forall v \in \mathrm{V}^{\ell}: v>1, \\
-\theta \leq \Delta_{v}^{\ell} \leq \theta, & \forall \ell \in \mathrm{L}, \forall v \in \mathrm{V}^{\ell} .
\end{array}
$$

Constraints (5) impose that the original cyclic departure times must respect the cycle. Constraints (6) set the maximum shift from the cyclic departure times. 0 min shifted cyclic timetable is equivalent to the cyclic one. For a cycle of one hour, $30 \mathrm{~min}$ shifted cyclic timetable denotes the highest possible deviation, otherwise the trains would overlap. Due to the case, where two consecutive trains might have +30 minutes and -30 minutes deviations, the constraints (6) have to be adjusted to the form:

$$
-\theta \leq \Delta_{v}^{\ell} \leq \theta-1, \quad \forall \ell \in \mathrm{L}, \forall v \in \mathrm{V}^{\ell} .
$$

Therefore, we test this type of timetable for all values of $\theta$ between 3 and 30 in 30 minute intervals.

$\xi$ Partially Cyclic Timetable This hybrid timetable is constructed by allowing a percentage of trains on a given line to be non-cyclic. The degree of the regularity is then expressed as $\xi \%$, where $\xi$ is a parameter decided a priori. Since different lines are served by a different amount of trains, applying the $\xi$ to each line separately might significantly disrupt the regularity of the service. Instead, we propose to treat the $\xi$ as a percentage of the number of trains of the most frequent line $\left(\max \left(\left|V^{\ell}\right|\right)\right)$ denoted as $\eta$. The $\eta$ number of trains of each line are having a cyclic departure time and the rest of the trains is having a non-cyclic departure time. The decision on which trains are to be cyclic and non-cyclic is arbitrary. Indeed, the order of appearance within the set $V^{\ell}$ does not have any impact. 
The optimal solution will consist in the same values of the departure times no matter the internal order of the trains. For a notation convenience, we assign the first $\eta$ trains to be cyclic.

$$
\mathrm{d}_{v}^{\ell}-\mathrm{d}_{v-1}^{\ell}=\mathrm{c} \cdot z_{v}^{\ell}, \quad \forall \ell \in \mathrm{L}, \forall v \in \mathrm{V}^{\ell}: 1<v \leq \eta=\max \left(\left|\mathrm{V}^{\ell}\right|\right) \cdot \frac{\xi}{100} .
$$

We test this timetable for all values of $\xi$ between $10 \%$ and $90 \%$ in $10 \%$ intervals. The $100 \%$ partially cyclic timetable is equivalent to the cyclic one and the $0 \%$ partially cyclic timetable is equivalent to the non-cyclic one. Note, that the $\xi$ of $0 \%$ is a special case. Since zero divided by any number is equal to zero, the above constraint does not hold. However, since the $0 \%$ partially cyclic timetable is equivalent to the non-cyclic one, it is sufficient to solve the non-cyclic version of the PCTTP. The non-cyclic trains are most likely to be scheduled within the high demand density periods of the day.

This hybrid timetable is inspired by Yang et al. (2010). They allow a certain number of lines to be non-cyclic. The motivation is again the infeasibility of the problem due to the high density of the trains.

Hybrid Cyclic Timetable Even though both, $\theta$ shifted cyclicity and $\xi \%$ partial cyclicity, keep some degree of regularity, the newly created patterns might be too disorganized for the passengers to see. Therefore we propose one additional type of a hybrid timetable. The hybrid cyclic timetable schedules non-cyclic trains only in the hours/cycles where there is already a cyclic train being scheduled. Note that according to constraints (7), not every cycle is required to have a cyclic train scheduled. With such pattern, all of the passengers would obtain the same level of service as a cyclic timetable, with more flexibility. Within a given cycle, the passenger can decide to use the cyclic train or the non-cyclic one (if there is any). The ratio, between the amount of cyclic and non-cyclic trains, is a decision of the model. To obtain such a timetable, the constraints (9)-(11) need to be included in the PCTTP model.

$$
\begin{array}{cl}
\mathrm{q}_{v}^{\ell} \cdot \mathrm{q}_{v^{\prime}}^{\ell} \cdot \mathrm{d}_{v}^{\ell}-\mathrm{q}_{v}^{\ell} \cdot \mathrm{q}_{v^{\prime}}^{\ell} \cdot \mathrm{d}_{v^{\prime}}^{\ell}=\mathrm{q}_{v}^{\ell} \cdot \mathrm{q}_{v^{\prime}}^{\ell} \cdot\left(\mathrm{c} \cdot \mathrm{z}_{v v^{\prime}}^{\ell}\right), & \forall \ell \in \mathrm{L}, \forall v, v^{\prime} \in \mathrm{V}^{\ell}: v>1, v \neq v^{\prime}, \\
\mathrm{y}_{\mathrm{k}}^{\ell} \leq \sum_{v \in \mathrm{V}^{\ell}: \mathrm{d}_{v}^{\ell} / \mathrm{c}=\mathrm{k}} \mathrm{q}_{v}^{\ell}, & \forall \mathrm{k} \in \mathrm{K}, \forall \ell \in \mathrm{L}, \\
\left(1-\mathrm{q}_{v}^{\ell}\right) \cdot \mathrm{d}_{v}^{\ell} \leq \mathrm{y}_{\mathrm{d}_{v}^{\ell} / \mathrm{c}}^{\ell} \cdot \mathrm{h}, & \forall \ell \in \mathrm{L}, \forall v \in \mathrm{V}^{\ell} .
\end{array}
$$

The cyclicity of a train is modeled through the binary decision $\mathrm{q}_{v}^{\ell}$. Value 1 indicates that the train is a cyclic one, 0 otherwise. The cyclicity pattern only among the departure times of the cyclic trains is enforced by the constraints (9). The planning horizon consists of $K=h / c$ cycles. For a planning horizon of one day, the value of $k$ is on the interval from 1 to 24 . Since the non-cyclic trains can be scheduled only in the cycles, where there is a cyclic train running, the binary decision $y_{k}^{\ell}$ indicates whether there is such a train in the cycle $k$ (equals to 1 ) or not (equals to 0). Since there is at most one cyclic train per cycle, the right hand side of constraints (10) is either eqaul to 1 (when there is a cyclic train scheduled in cycle k) or equal to 0 (otherwise). Lastly, the constraints (11) allow for non-cyclic trains to exist only in the cycles, where there is a cyclic train scheduled. Note that the constraints (9)-(11) introduce non-linearity in the model. 


\section{Solution Methodology}

We use the Simulated Annealing (SA) heuristic to solve the PCTTP. Since the SA is a well known heuristic (defined by Kirkpatrick et al. (1983)), we focus only on the description of the specifics of the heuristic with respect to the PCTTP. The general pseudocode of the heuristic is shown in Algorithm 1.

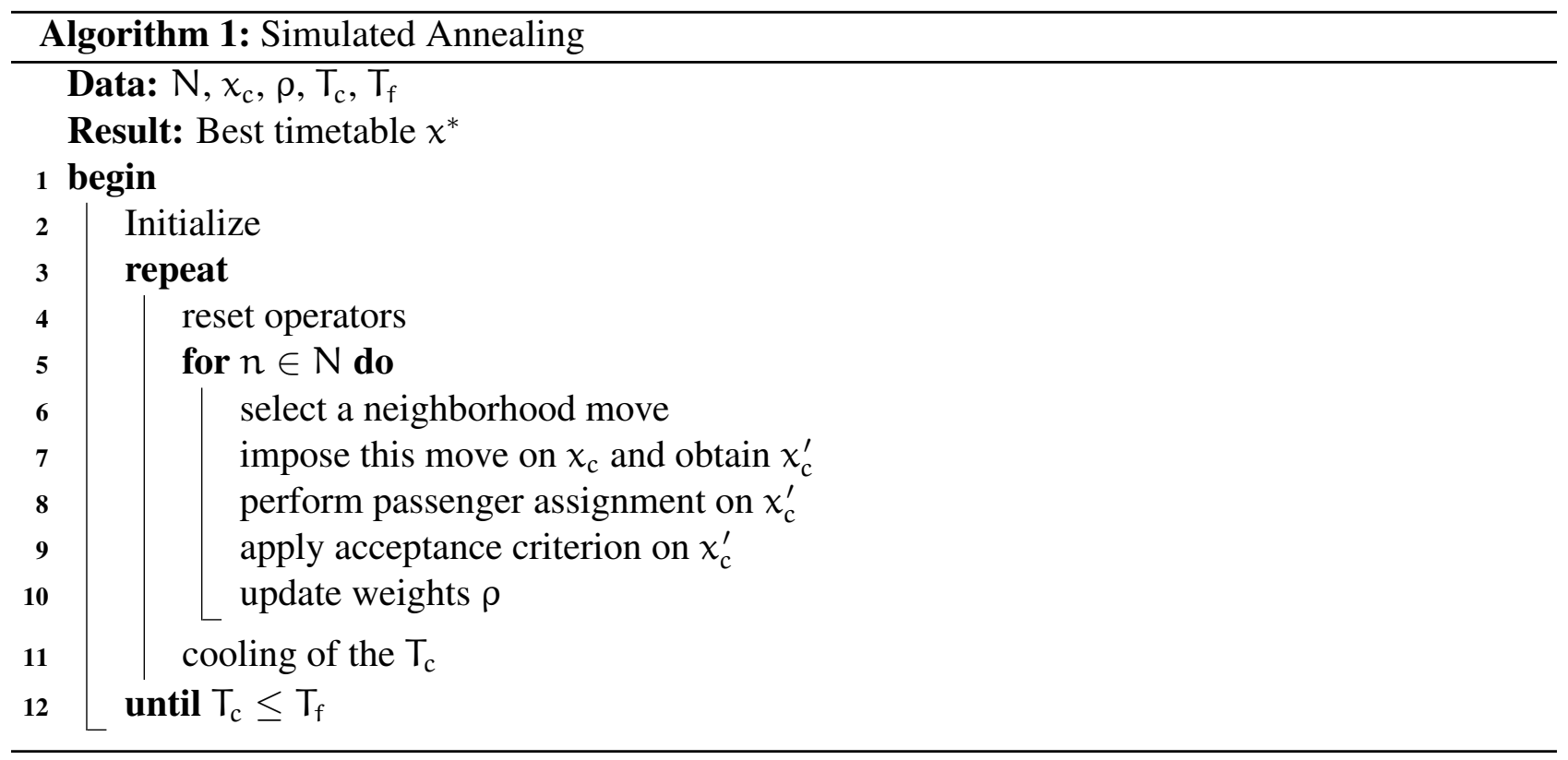

\subsection{Solution Representation}

A solution of the PCTTP problem consists in the departure times $d_{v}^{\ell}$ for every train $(\ell, v)$, that constitute the timetable, and its underlying flexibility. We distinguish among four types of a solution: the initial solution, the current solution $x_{c}$, the potential new solution $x_{c}^{\prime}$ and the best found solution $x^{*}$. Any cyclic timetable can be used as an initial solution. Indeed, the other types of timetable have less constraints, so that a cyclic timetable verifies their constraints as well. A cyclic timetable can be constructed for instance by randomly generating the departure time of the first train of every line and setting the departure times of every subsequent train on every line by adding the value of the cycle to the departure time of the previous train. The other three types of a solution follow the standard SA logic.

The flexibility of a solution is its passenger satisfaction that is estimated using the value function (see Section 6.2). 


\subsection{Value Function And Its Estimation}

The value function of the problem is the overall passenger satisfaction that is to be maximized. In order to estimate its value, the passenger assignment to the trains is carried out on the associated timetable. We design our own passenger assignment procedure. The typical assignment in the literature is not suitable for our value function. The classical assignment approach is based on the first come first serve (FCFS) policy. Such an assignment provides user optimum whereas the goal of the PCTTP is the global optimum. Moreover, since the demand elasticity is not modeled, the FCFS might give priority to a passenger that might not realize her journey and a passenger that would is either left out or re-routed on a worse path due to the capacity issues. Whereas in the PCTTP, the goal is to take care of the demand elasticity implicitly by maximizing the overall passenger satisfaction.

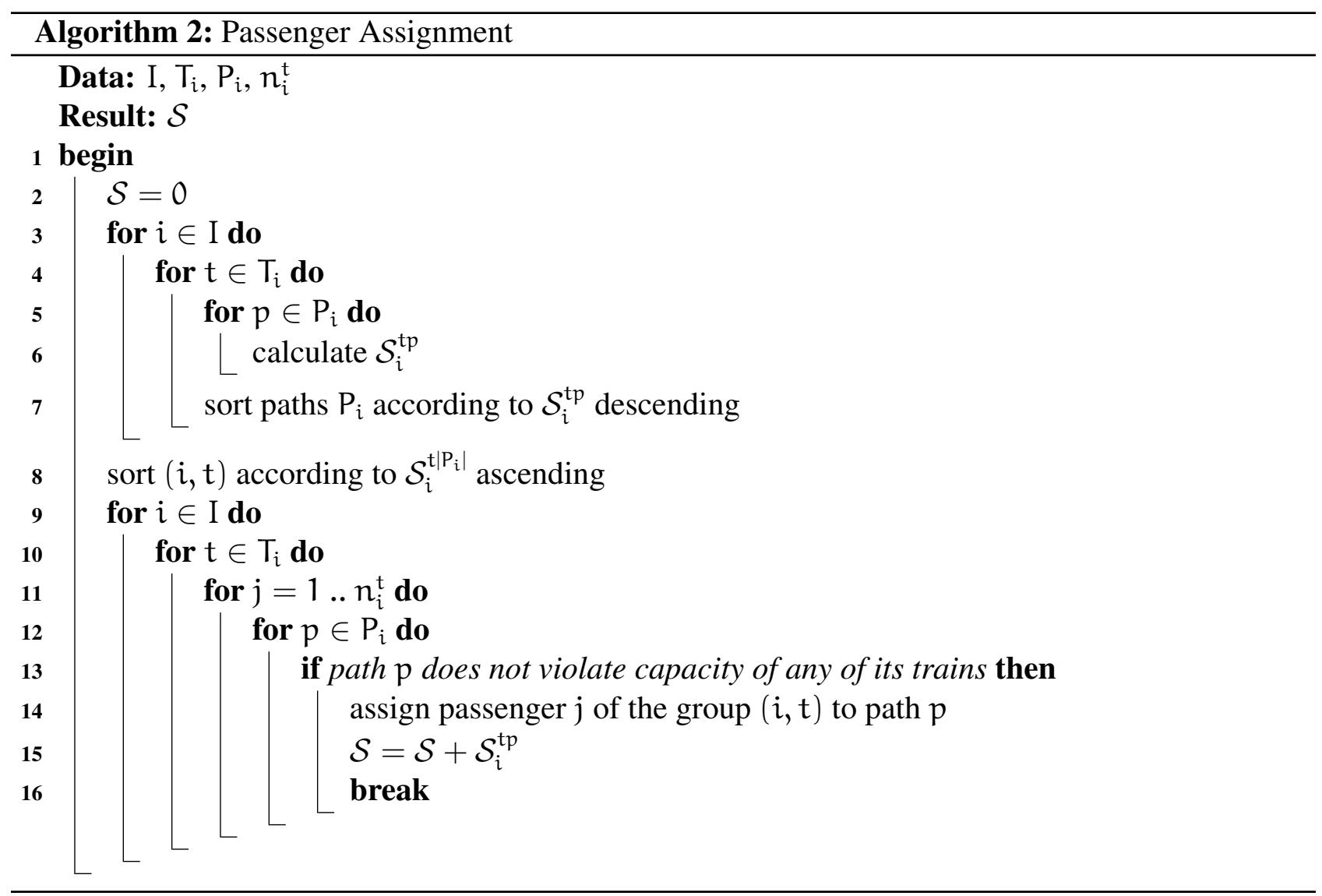

The general pseudocode of the proposed passenger assignment is shown in Algorithm 2. In the first stage of the algorithm, the satisfaction of every path of each of the passenger groups is calculated and sorted in the descending order. This part of the algorithm is parallelized. In the second stage of the algorithm, the passenger groups are sorted according to their last (worst) path (index $\left|\mathrm{P}_{i}\right|$ ) in the ascending order. This means that, in the next stage, the first processed passenger is having the worst possible satisfaction of the whole problem within her choice set. However, since the paths are sorted in the descending fashion, she will be first offered her best possible path. The algorithm splits 
the passenger groups into individuals, in order to achieve better values of the overall satisfaction. For each of the passengers, the algorithm iterates through the respective set of possible paths and assigns the first realizable path. Such a path does not violate the capacity constraints of the trains. No passenger remains unassigned as each set $P_{i}$ contains the respective penalty path. The algorithm terminates once all of the passengers are assigned to the paths.

\subsection{Neighborhood Structure}

Each type of a timetable is having a different neighborhood structure. A neighborhood is defined as a set of candidate solutions that can be reached by a modification of the current solution $x_{c}$ using a single neighborhood move. A timetable type can have more than one neighborhood that is characterized by its move. An overview of all the moves per type of a timetable is shown in Table 2.

\begin{tabular}{|c|c|c|c|c|c|c|c|}
\hline & & N. & Cyclic & $\theta$ Shifted C. & そ Partially C. & Hybrid Cyclic & Distribution \\
\hline \multirow{4}{*}{ Select } & $(\ell, v)$ & & & & & & $\mathcal{U}(1,|\mathrm{~L}|), \mathcal{U}\left(1,\left|V^{\ell}\right|\right)$ \\
\hline & $\ell$ & & & & & & $\mathcal{U}(1,|\mathrm{~L}|)$ \\
\hline & $q_{v}^{\ell}=0$ & & & & & & \\
\hline & $\mathrm{q}_{v}^{\ell}=1$ & & & & & & \\
\hline \multirow{4}{*}{ Modify } & $\mathrm{d}_{v}^{\ell}$ & & & & & & $\mathcal{U}(0, h-1)$ \\
\hline & $\mathrm{d}_{v}^{\ell} \bmod \mathrm{c}$ & & & & & & $\mathcal{U}(0, c-1)$ \\
\hline & $k$ & & & & & & $\mathcal{U}(0, \mathrm{~h} / \mathrm{c}-1)$ \\
\hline & $\Delta_{v}^{\ell}$ & & & & & & $\mathcal{U}(-\theta, \theta)$ \\
\hline \multirow{3}{*}{ Apply } & $\forall \mathbf{q}_{v}^{\ell}=1$ & & & & & & \\
\hline & $y_{k / c}^{\ell}=0$ & & & & & & \\
\hline & $\mathrm{y}_{\mathrm{d}_{v}^{\ell} / \mathrm{c}}^{\ell}=1$ & & & & & & \\
\hline
\end{tabular}

Table 2: Overview of the neighborhood moves by the type of a timetable

The columns represent the moves and the rows represent a stage of a move. The moves are categorized by the type of a timetable they belong to. The first column denoted N. stands for the non-cyclic timetable. The last column shows the distributions from which to draw randomly, in order to obtain the respective attribute's value. Only the cells with a grey background constitute a move. Each move is decomposed into 3 parts: selection, modification and application. Two entities can be selected: a specific train $(\ell, v)$ or the whole line $\ell$. The selection of a specific train might be conditioned by its type $\left(q_{v}^{\ell}\right)$. If no condition is specified, any train can be selected.

The aim of the modification is to replace the value of one of the 4 attributes with a new one. The only not yet defined attribute is $\mathrm{d}_{v}^{\ell}$ modc. This attribute represents the modulo time within any cycle. For instance, when the cycle $c$ is equal to one hour and the departure time of a train is $5: 45$, then the modulo time is 45 minutes. This time is the same among all cyclic trains of the same line.

The application of the modification is always performed on the pre-selected entity. However, its application might be conditioned. Some applications are made only to the cyclic trains of the given 
line $\left(\forall q_{v}^{\ell}=1\right)$. Other applications are conditioned that the new cycle $k$ does not already contain a cyclic train $\left(y_{\mathrm{k} / \mathrm{c}}^{\ell}=0\right)$ or that the new departure time $\mathrm{d}_{v}^{\ell}$ is in a cycle that does already contain a cyclic train $\left(y_{\mathrm{d}_{v}^{\ell} / \mathrm{c}}^{\ell}=1\right)$. If the newly generated values of attributes fail to fulfil the last two conditions (when they are required), the modification is repeated (until they do).

For illustration, consider the first move of the hybrid cyclic timetable: at first a train is selected by drawing the line $\ell$ from $\mathcal{U}(1,|\mathrm{~L}|)$ and subsequently drawing the $v$ from $\mathcal{U}\left(1,\left|\mathrm{~V}^{\ell}\right|\right)$. The drawing from the distribution(s) is repeated until the condition $q_{v}^{\ell}=0$ is satisfied. Hence, a non-cyclic train has been selected. In the second phase, a new departure time $d_{v}^{\ell}$ of this train is drawn from $\mathcal{U}(0, h-1)$. In the third phase, this new departure time is applied given that it is in the cycle where there is a cyclic train scheduled $\left(y_{\mathrm{d}_{v}^{\ell} / \mathrm{c}}^{\ell}=1\right)$. Otherwise the modification phase is repeated until this condition is complied.

When a timetable type has more than one neighborhood move $j$, each of the moves is associated with a weight $\rho_{j}$. Each $\rho_{j}$ is initialized to 1 at the beginning of every new temperature $T_{c}$. Based on the performance of the move, its weight is being updated according to the below scheme $(f(x)$ is the value function):

- If $f\left(x_{c^{\prime}}\right) \geq f\left(x^{*}\right) \rightarrow \rho_{j}=\rho_{j}+3$

- If $f\left(x_{c^{\prime}}\right) \geq f\left(x_{c}\right) \rightarrow \rho_{j}=\rho_{j}+2$

- If $f\left(x_{c^{\prime}}\right)<f\left(x_{c}\right)$ and the new solution has been accepted with a probability $r<\exp \left(-\left(x_{c}-\right.\right.$ $\left.\left.x_{c^{\prime}}\right) / T_{c}\right)$, where $r$ is drawn from $\mathcal{U}(0,1)$, then $\rho_{j}=\rho_{j}+1$

In each iteration of the SA heuristic, a move is selected using the roulette wheel mechanism based on the weights $\rho$ of the currently solved type of a timetable.

\subsection{Values Of The Parameters}

We set the value of the final temperature $T_{f}$ to be zero and the initial temperature $T_{0}$ to be a function of the passenger satisfaction value of the initial timetable $f\left(x_{0}\right)$ where $T_{0}=10^{-5} \cdot f\left(x_{0}\right)$. The other two parameters, which values need to be tested, are the cooling scheme and the total number of iterations $\mathrm{N}$ per temperature $\mathrm{T}_{\mathrm{c}}$. The cooling scheme provides the information on how to decrease the temperature $T_{c}$. We test the cooling schemes of 5,10 and $20 \%$ of the initial temperature $T_{0}$ and the values of $\mathrm{N}$ of 100,500 and 1000 iterations.

The tests were performed on 5 realistic instances of the S-train network of Canton Vaud in Switzerland as desribed by Robenek et al. (2016) for cyclic and non-cyclic timetables. As the rest of the timetables uses a combination of the neighborhood moves contained in the two timetables, there is no need for an explicit testing of the other types. We have performed 10 runs of each combination of the parameter settings for both types of the timetables. The cooling scheme of $5 \%$ and the number of iterations $N=1000$ provided on average the best values of the passenger satisfaction. Given that the solution time was fast (on average 5 minutes for both cyclic and non-cyclic timetables), we have selected it as our final parameter setting. 


\subsection{Validation}

In this section, we validate the SA algorithm as compared to CPLEX on a small artificial network. The considered instances vary in the distribution and the number of passengers, and the capacity of the trains. The precise information of each of the instances is incorporated in their names: 0 - if the demand was evenly distributed across the planned horizon (the horizon is 3 hours), 1 - otherwise; $d$ - provides the total number of the passengers in the network; $\mathrm{c}$ - is the capacity of a single train (in total there are 16 trains).

The detailed numerical results can be found in Table 3. They have been categorized by the type of the solved timetable: cyclic or non-cyclic. The first column provides the information about the solved instance. The optimal solution is the passenger satisfaction obtained by CPLEX and SA is the passenger satisfaction obtained by the SA heuristic. Both values are in Swiss Francs. All the methods have solution time reported in seconds. The gap between the optimal solution and the solution found by the SA heuristic is reported in percentages.

\begin{tabular}{|c|c|c|c|c|c|c|c|c|c|c|}
\hline \multirow[b]{2}{*}{ Instance } & \multicolumn{5}{|c|}{ Cyclic Timetable } & \multicolumn{5}{|c|}{ Non-Cyclic Timetable } \\
\hline & Optimal & Time $[\mathrm{s}]$ & SA & Time[s] & Gap [\%] & Optimal & Time $[\mathrm{s}]$ & SA & Time[s] & Gap [\%] \\
\hline $0-\mathrm{d} 35-\mathrm{c} 1$ & -3269 & 4 & -3358 & 11 & 2.72 & -3208 & 2 & -3272 & 12 & 1.98 \\
\hline $0-\mathrm{d} 41-\mathrm{c} 2$ & -2504 & 59 & -2624 & 13 & 4.80 & -2482 & 127 & -2589 & 12 & 4.29 \\
\hline $0-\mathrm{d} 40-\mathrm{c} 2$ & -2515 & 385 & -2570 & 14 & 2.20 & -2479 & 152 & -2507 & 10 & 1.15 \\
\hline $1-d 37-c 2$ & -2184 & 18 & -2236 & 11 & 2.37 & -2165 & 40 & -2215 & 11 & 2.30 \\
\hline $0-\mathrm{d} 53-\mathrm{c} 3$ & -3729 & 6177 & -3887 & 13 & 4.25 & -3698 & 716 & -3826 & 13 & 3.45 \\
\hline $1-\mathrm{d} 36-\mathrm{c} 3$ & -3020 & 4 & -3066 & 11 & 1.50 & -2989 & 3 & -3019 & 11 & 0.99 \\
\hline $0-\mathrm{d} 52-\mathrm{c} 4$ & -5686 & 20 & -5761 & 12 & 1.32 & -5641 & 7 & -5720 & 13 & 1.41 \\
\hline 1-d63-c4 & -6568 & 2686 & -6656 & 14 & 1.34 & -6531 & 2905 & -6613 & 14 & 1.26 \\
\hline 1-d63-c4 & -6854 & 6853 & -6966 & 12 & 1.63 & -6803 & 1854 & -6904 & 14 & 1.49 \\
\hline $1-\mathrm{d} 80-\mathrm{c} 4$ & -11204 & 1457 & -12021 & 15 & 7.29 & -11133 & 32 & -11866 & 15 & 6.58 \\
\hline Avg. & -4753 & 1766 & -4914 & 13 & 2.94 & -4713 & 584 & -4853 & 13 & 2.49 \\
\hline
\end{tabular}

Table 3: Validation of SA as compared to the optimal solutions

The SA heuristic can find solutions with a reasonable gap from the optimal solution. The worst performance is for the instances $0-d 41-c 2,0-d 53-c 3$ and $1-d 80-c 4$. The first two instances are hard to solve as the demand is almost matching the supply. Therefore the order, in which the passengers are being processed in the assignment procedure, plays a crucial role. The FCFS policy would obtain worse values. The instance 1-d80-c4, on the other hand, has the most extreme passenger distribution: $90 \%$ of the passengers in the first hour and $5 \%$ in each of the two subsequent hours. The average gap for the cyclic timetable is $2.94 \%$ and for the non-cyclic timetable $2.49 \%$. In terms of the time, the SA heuristic performs better than CPLEX.

\section{Case Study}

In order to evaluate the performance of the various timetables, we apply the proposed methodology on the network of Israeli Railways (IR) shown in Figure 1. The aim is to compare the level of passenger 
satisfaction for different timetables. The exact procedures, assumptions and information about the data can be found in Appendix A.

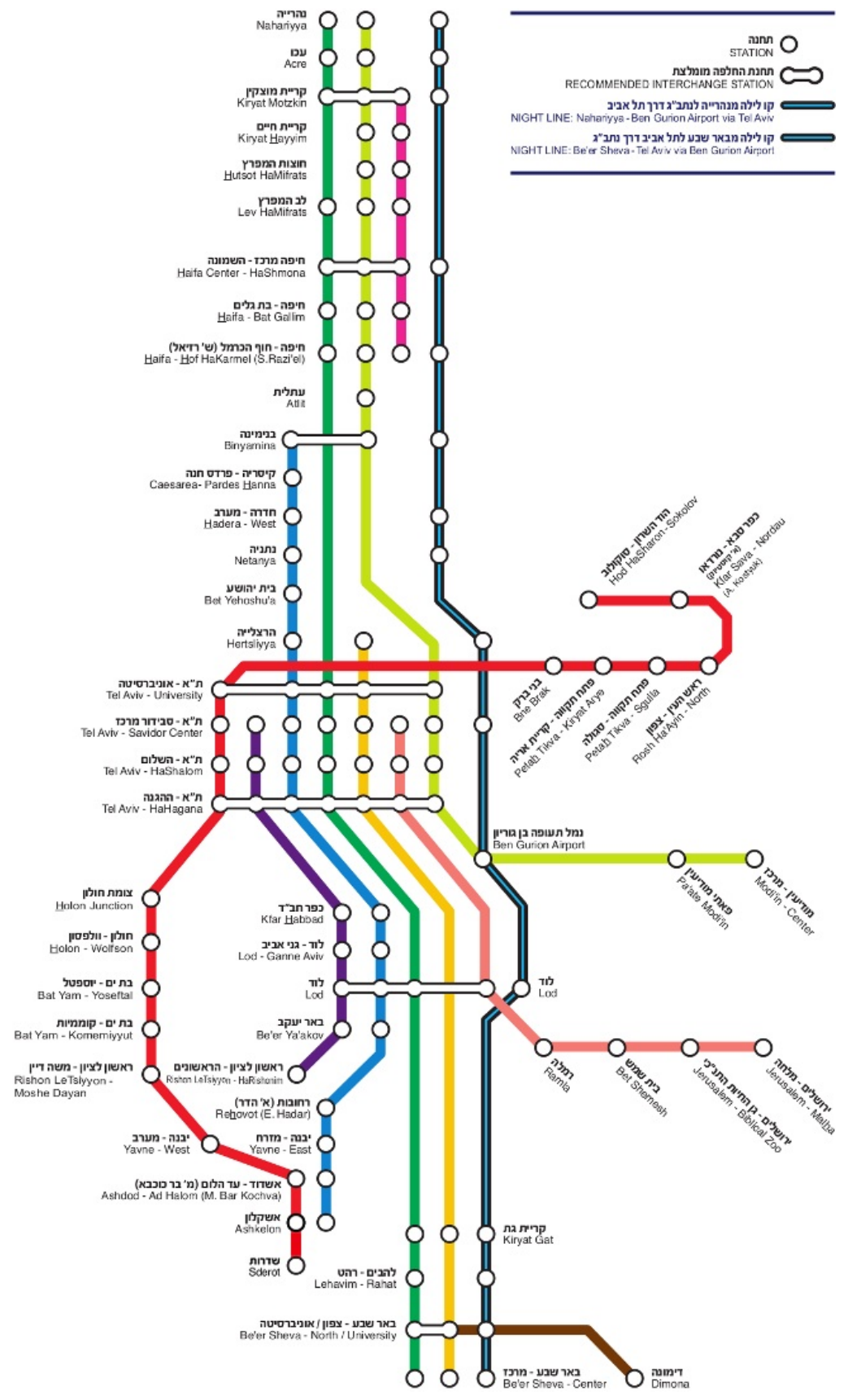

Figure 1: Network of Israeli Railways (www.rail.co.il)

We consider two instances in our study: the 2008 demand and the 2014 demand. The 2008 demand is build from the ticket selling machines' data of an average working day (from 6 a.m. to 1 a.m.) in 2008 in Israel. This data was kindly provided to us by Mor Kaspi and Tal Raviv, who have used it in 
their study Kaspi and Raviv (2013). The 2008 demand constitutes in 126036 passengers. The 2014 demand constitutes in 193886 passengers and it has been created from the 2008 demand multiplied by the constant factor of 1.6 per OD pair (based on the newspaper article in Globes (2015)).

The demand data is available for the year 2008. Therefore, we consider the network layout of 2008: there were 47 stations and the red line in Figure 1 was operated only between Hod HaSharon and Tel Aviv - HaHagana. We do not consider the night line (blue with black bordering) as it runs mainly in the period for which we do not have the demand data (i.e. between 1 a.m. and 6 a.m.). Even though there are only 18 unidirectional lines visualized in Figure 1, in reality there are 34 unidirectional lines in the timetable (some lines are operated with higher frequency of 2 or more modulo times). Since some of the trains follow different stopping patterns within a line, we have taken a union of stopping stations for each line (but same colored lines with different modulo times may operate different stopping patterns). Since the OD matrix is given for an average working day, we have removed the trains that operate only during the holidays.

The timetable operated in Israel is cyclic with a cycle of 60 minutes. We have processed the timetable of 2013/14 ${ }^{1}$ where 6 out of 388 trains have non-cyclic departure times. The planning horizon $h$ of this case study is one day. Even though the demand is between 6 a.m. and 1 a.m., we allow the PCTTP model to schedule trains during any time of the day. We solve the PCTTP using the SA heuristic for the following timetables:

- Non-Cyclic - no specific rule on the departure times is enforced.

- Cyclic - the departure times have to be cyclic according to the constraints (3).

- $\theta$ Shifted Cyclic - the departure times are subject to the constraints (5) and (6). The values of $\theta$ vary between 3 and 30 in 3 minute intervals. Since $\theta$ of 0 is equivalent to the cyclic timetable, it does not need to be solved.

- $\xi$ Partially Cyclic - the departure times are subject to the constraints (8). The values of $\xi$ vary between 10 and 90 in 10\% intervals. Since $\xi$ of 0 and 100 is equivalent to the cyclic and the non-cyclic timetables respectively, they do not need to be solved.

- Hybrid Cyclic - the departure times have to comply with the constraints (9) - (11).

For the sake of the comparison, we show the performance of the two below timetables as well:

- IR 13/14 - the departure times are fixed to the ones of IR timetable of 13/14. The IR timetable is cyclic as using the constraints (3), with an exception of having the aforementioned 6 noncyclic trains.

- IR 13/14 as Strictly Cyclic - is the same as IR 13/14, where the 6 non-cyclic trains are fixed to their closest cyclic departure time, and the gaps between the first and the last scheduled train of every line are filled with cyclic trains (in total 82 trains more). This is equivalent to having the constraints (2). The exact difference between the two timetables can be observed on the train distributions in Table 7 and Table 8 in Appendix B.

\footnotetext{
${ }^{1}$ Unlike Europe, the timetable change in Israel happens during the summer period, i.e. the naming 13/14.
} 
The passenger satisfaction of each type of timetable is given in New Israeli Shekels (NIS). All of the tested instances have been run in Java on a Unix server with up to 24 cores of $3.33 \mathrm{GHz}$ and 62 GiB RAM. Since we would typically run 3 instances at the same time, the average core usage would be then 8 .

\subsection{Results}

In this section, we present the results of our case study. We categorize the results into two groups: existing and hybrid timetables. The existing ones are: strictly cyclic, cyclic and non-cyclic timetables. The hybrid ones are: $\theta$ shifted cyclic, $\xi$ partially cyclic and hybrid cyclic timetables.

At first, we construct the demand distributions over the day (Figure 2). Each distribution is in fact a network load, where the passengers take their shortest path between their origin and destination with just a minimum transfer time (i.e. no waiting time in transfers) and arriving to their destinations exactly on time, thus their schedule passenger delay being zero. Note that this network load does not involve any actual timetable and it is only used as a benchmark. We denote it as the perfect service.

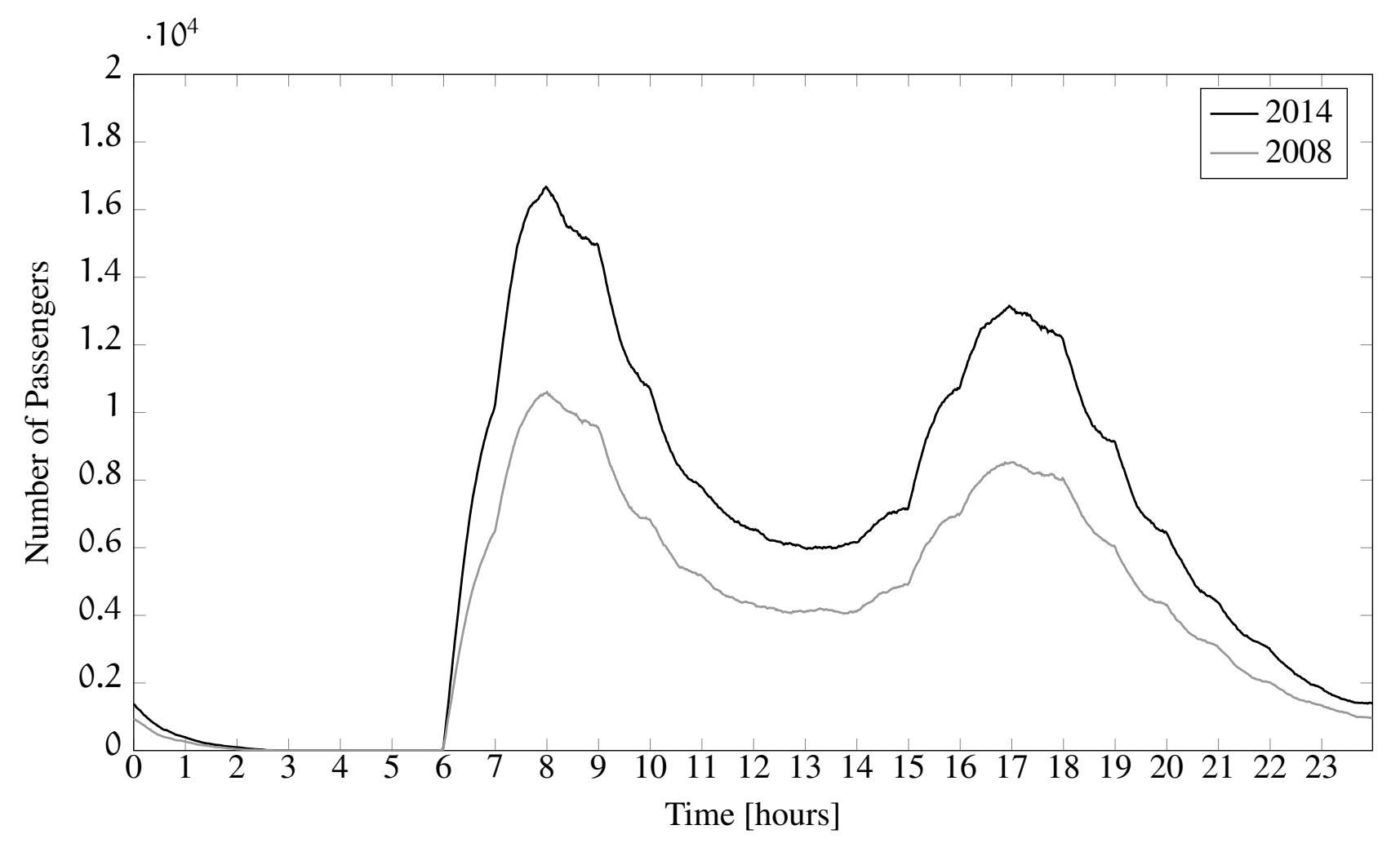

Figure 2: Demand distributions over the day

The passenger satisfaction of the 2008 demand distribution under the perfect service is -2089049 NIS and of the 2014 demand distribution -3 171721 NIS. These two values are upper bounds of the two instances for all the types of the timetables (no better solution exists). The aim of the timetable design should be a network load as close as possible to the perfect service. 


\subsubsection{Existing Timetables}

The performance of each timetable can be found in Table 4 for the 2008 demand and in Table 5 for the 2014 demand. For each timetable, we report the underlying passenger satisfaction in New Israeli Shekels (NIS). The perfect service reports the satisfaction as it is and the different timetables report the passenger satisfaction as a relative difference to the perfect service. Apart from the satisfaction, the tables provide information on the number of train drivers needed to realize the operation, as well as the needed number of train units, the percentages of passengers that were able to realize their journeys and the solution time in seconds.

\begin{tabular}{r|ccccc}
\hline & IR 13/14 as Strictly Cyclic & IR 13/14 & cyclic & non-cyclic & perfect service \\
\hline satisfaction [NIS] & -704904 & -537503 & -476774 & -424529 & -2089049 \\
drivers [-] & 470 & 388 & 388 & 388 & 48960 \\
rolling stock [-] & 940 & 776 & 776 & 776 & 48960 \\
covered [\%] & 100 & 100 & 100 & 100 & 100 \\
time [sec] & 12 & 6 & 24997 & 25613 & 1 \\
\hline
\end{tabular}

Table 4: Computational results of the existing timetables for the 2008 demand

\begin{tabular}{r|ccccc}
\hline & IR 13/14 as Strictly Cyclic & IR 13/14 & cyclic & non-cyclic & perfect service \\
\hline satisfaction [NIS] & -3792733 & -3379596 & -2392909 & -1365779 & -3171721 \\
drivers [-] & 470 & 388 & 388 & 388 & 48960 \\
rolling stock [-] & 940 & 776 & 776 & 776 & 48960 \\
covered [\%] & 99.17 & 99.32 & 99.32 & 99.23 & 100 \\
time [sec] & 11 & 8 & 86627 & 88342 & 2 \\
\hline
\end{tabular}

Table 5: Computational results of the existing timetables for the 2014 demand

It is interesting to see (from the tables) that even though the IR 13/14 as strictly cyclic timetable offers more trains, its passenger satisfaction is lower than of the IR 13/14 timetable for both demand distributions. The reason behind this, is the fact that there are 6 non-cyclic trains in the IR 13/14 timetable that need to have a cyclic departure time in the strictly cyclic one. The decrease is then caused by the additional waiting time between the original non-cyclic departure and the new (strictly) cyclic one. Overall, running a strictly cyclic timetable is not desirable as the operating cost is higher and the additional benefit is either low or non-existent.

When we compare the IR 13/14 timetable with the cyclic timetable, we can see that proportionally large increase in passenger satisfaction can be achieved: approx. 60000 NIS for the 2008 demand and approx. 1 million NIS for the 2014 demand. As the passenger coverage is $100 \%$ and $99.32 \%$ respectively, we can assume that the 2014 demand is just about the maximum capacity of the network, whereas the 2008 demand is over supplied. 
A similar situation happens for the difference between the cyclic and the non-cyclic timetable. The differences in the passenger satisfaction are approx. 50000 NIS and approx. 1 million NIS for the two demand distributions respectively. The solution time of the SA heuristic is on average 7 hours for the 2008 demand and one day for the 2014 demand. As the differences among the timetables in the 2008 demand were marginal, we will from now on keep our focus only on the 2014 demand.

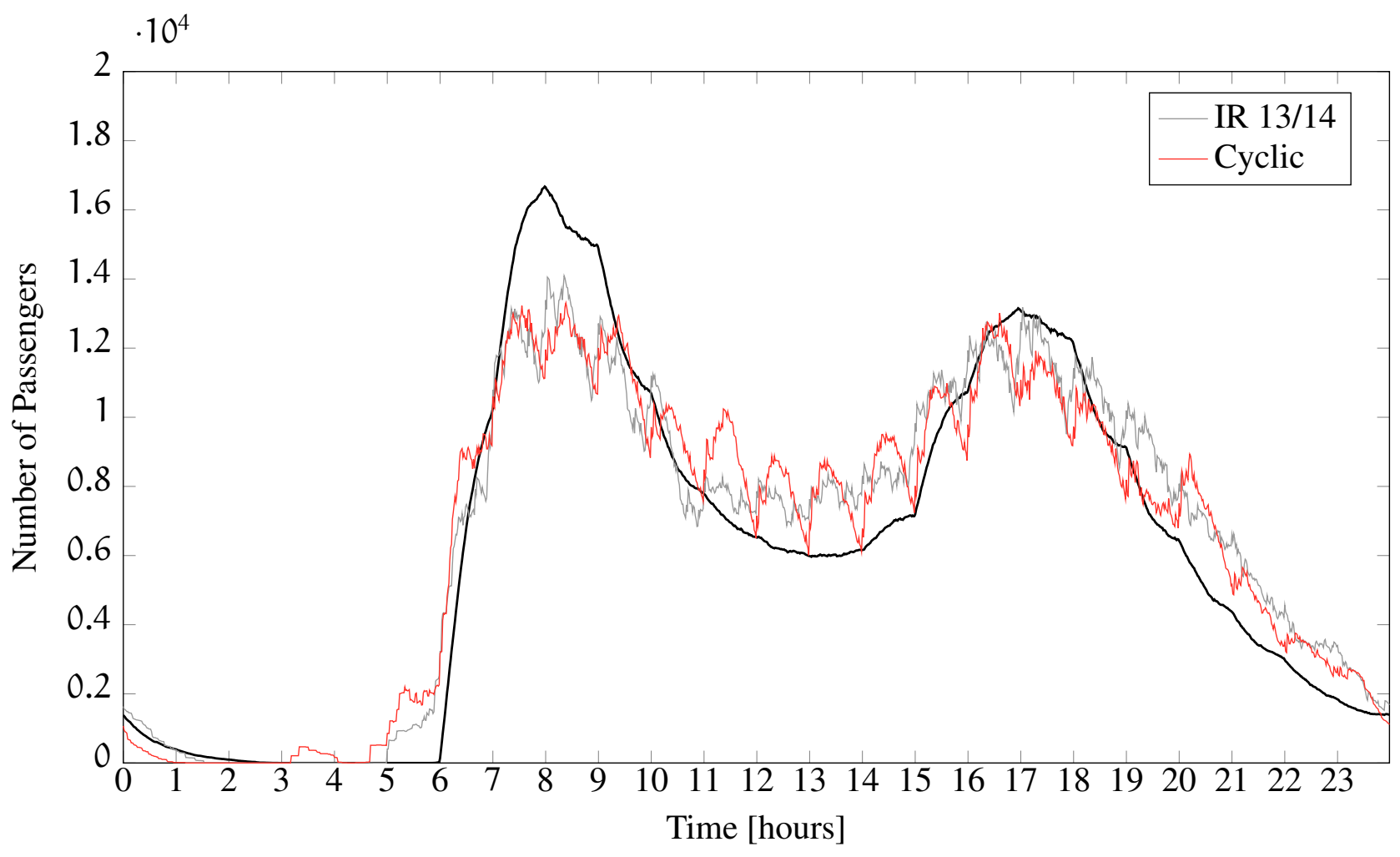

Figure 3: Network load of the IR 13/14 and of the cyclic timetable for the 2014 demand

When we plot the network load for the 2014 case of the IR 13/14 timetable and of the cyclic timetable (Figure 3), we can see that both timetables are fairly good in addressing the evening peak hours, but fail at addressing the morning ones. The failure propagates into surrounding time periods: early start of the morning peak (i.e. some passengers arriving early at their destinations) and a heightened level of passengers in the network in between the peak hours (i.e. late arrivals from the morning peak and early arrivals for the evening peak). Both timetables exhibit the nature of the repeating pattern inside the plot.

The improvement of the cyclic timetable over the IR 13/14 timetable is approx. 1 million NIS. According to the breakdown of the passenger satisfaction (in Figure 5), the improvement is mainly in the passenger schedule delay. This is due to the fact, that the cyclic timetable is having more passengers arriving early in to their destinations than the IR 13/14 timetable. Indeed, the $\beta$ estimate of being early has lower value $(=0.5)$ than the $\beta$ estimate of being late $(=1)$.

When we compare the network load of the cyclic and the non-cyclic timetable (Figure 4), we can see that the non-cyclic one is indeed more flexible in accounting for the passenger demand. Especially 


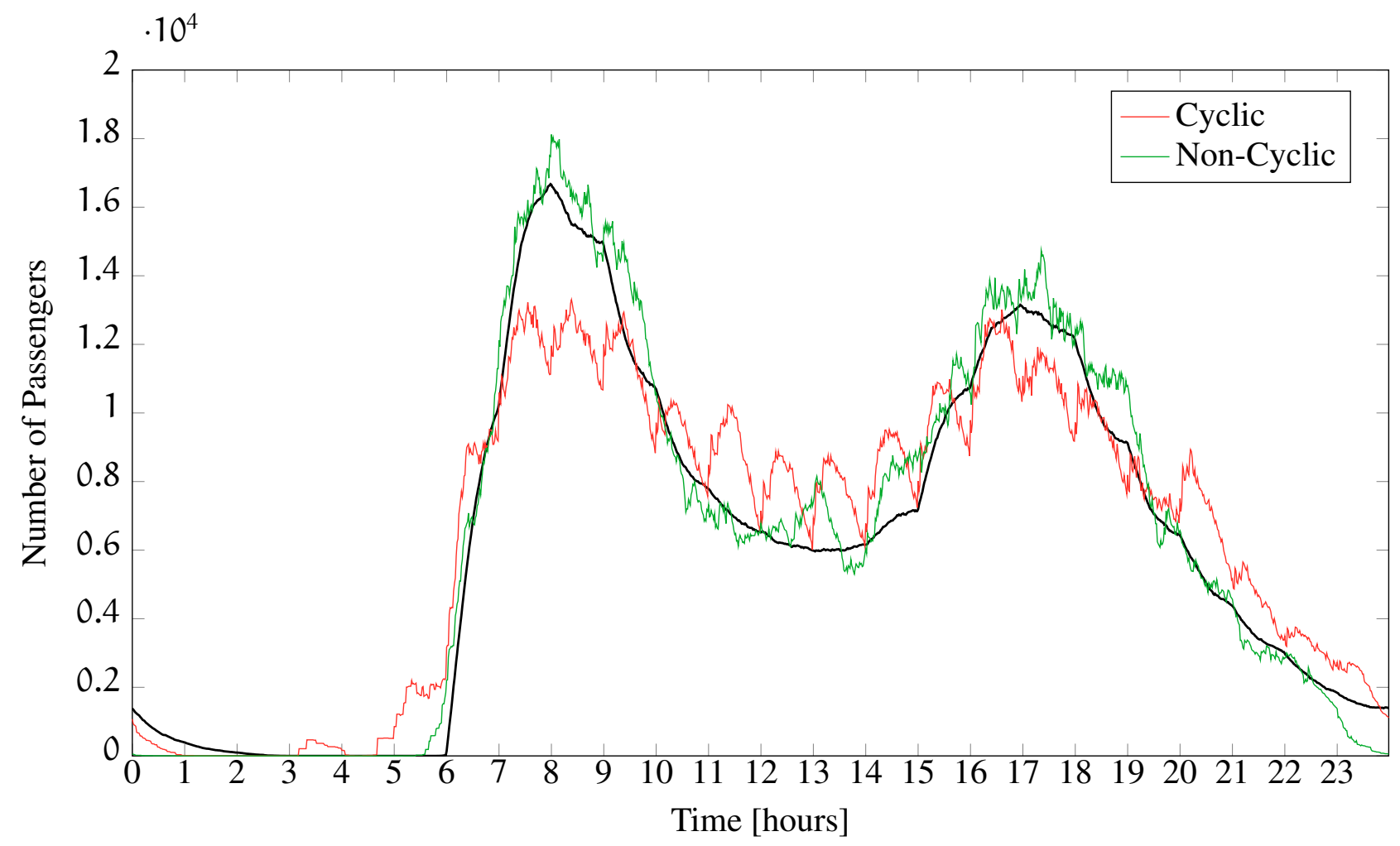

Figure 4: Network load of the cyclic and of the non-cyclic timetable for the 2014 demand

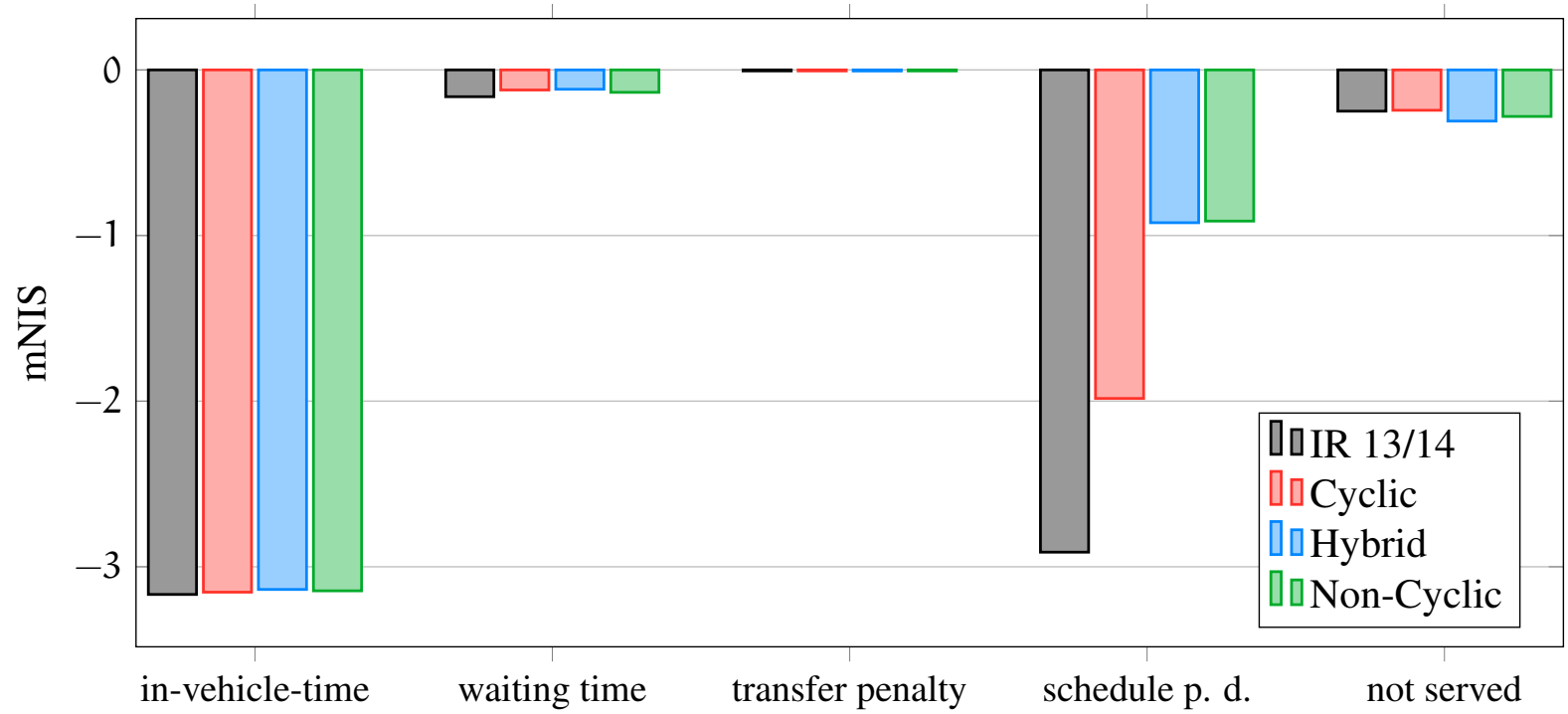

Figure 5: Breakdown of the passenger satisfaction for various timetables under the 2014 demand

within the peak hours, where there is often more than one train being scheduled (in some cases even 4 trains per hour, Table 10 in Appendix B). This brings about one more million NIS of improvement 
in the schedule passenger delay (Figure 5).

When we look at the breakdown of the passenger satisfaction (Figure 5), we can see that the most important is the attribute of the schedule passenger delay representing the fact that the passenger demand is time dependent. Thus, when designing a railway timetable, one should always take into account the passengers and their travel time preferences.

\subsection{2 $\theta$ Shifted Cyclic Timetable}

As mentioned before, we solve this timetable for various values of $\theta$ within the interval between 3 and 30 in 3 minute step size ( 0 being the solution of the cyclic timetable). The plot of the passenger satisfaction as a function of $\theta$ can be found in Figure 6.

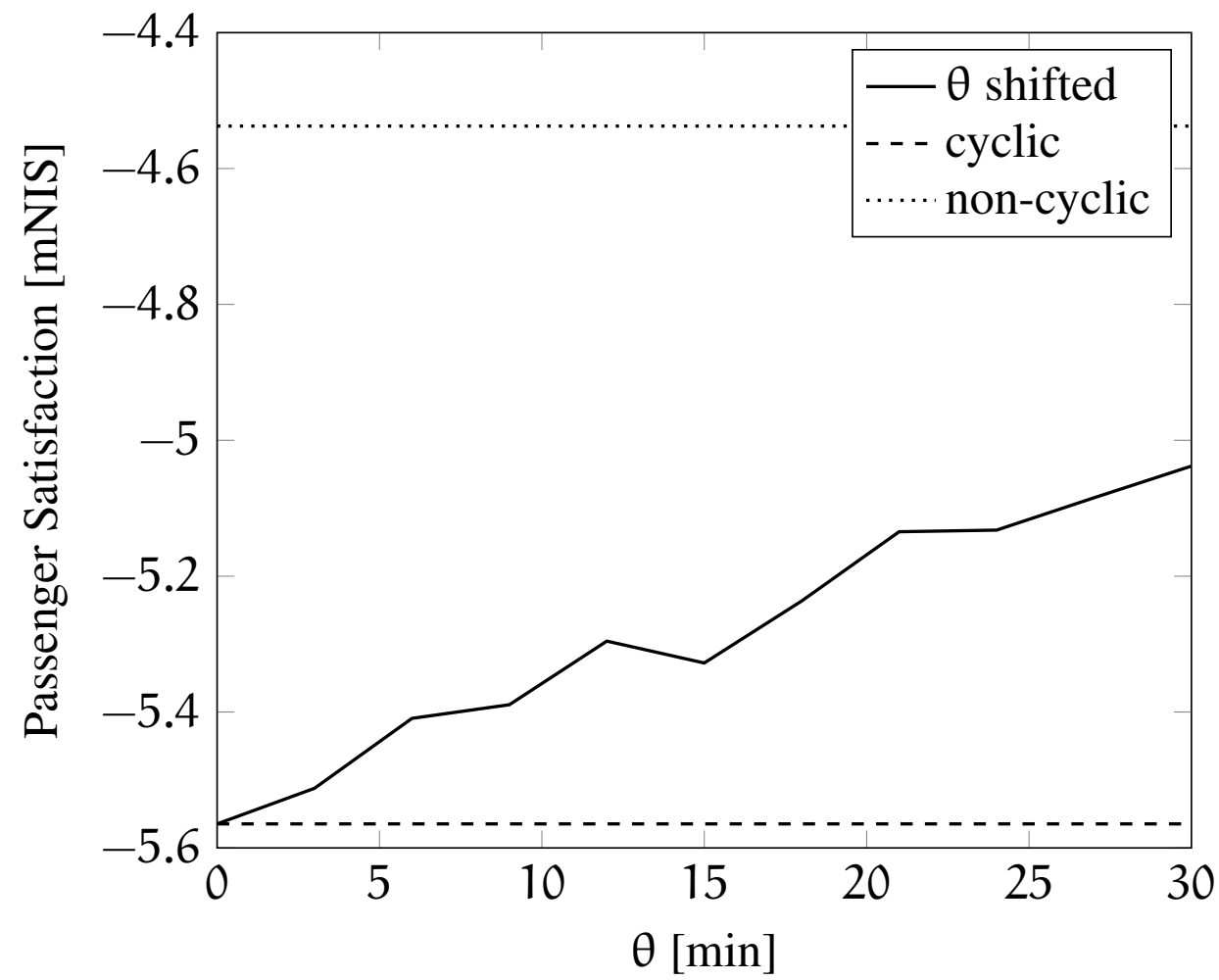

Figure 6: Passenger satisfaction of the $\theta$ shifted cyclic timetable for the 2014 demand

The 0 min shifted cyclic timetable is equivalent to the cyclic one, which is represented by the dashed line. The 30 min shifted cyclic timetable, on the other hand, is not equivalent to the non-cyclic one (the dotted line), as the maximum number of trains that are scheduled within one hour is 2 (Table 11 in Appendix B) and 4 (Table 10 in Appendix B) respectively. Thus the $\theta$ shifted cyclic timetable can achieve at most half of the flexibility of the non-cyclic one (around half a million NIS). The trend of the function is rather linear.

Overall, the improvement in flexibility of the newly proposed timetable is promising. It is a sign that new alternative timetables exist and should be taken into account. As this was the more restrictive 
configuration, we expect even larger impacts from the other two hybrid timetables.

\subsection{3 $\xi$ Partially Cyclic Timetable}

In this section, we present the computational results of the $\xi$ partially cyclic timetable for different levels of partiality under the 2014 demand (Table 6). The first column represents the level of the partiality as a percentage. The second column shows the passenger satisfaction in NIS. The satisfaction for $\xi=100$ is given as it is and all other satisfactions are given as relative to this one. Next columns give information on the $\eta$ (which is the number of trains per line that have to follow the cyclic pattern, Equation 8), the total number of trains that follow cyclic pattern, the total number of trains that are non-cyclic and the solution time of the SA heuristic.

\begin{tabular}{r|rcccc}
\hline$\xi[\%]$ & P. Satisfaction [NIS] & $\eta$ & Cyclic & Non-Cyclic & Time[sec] \\
\hline 0 & +1027131 & 0 & 0 & 388 & - \\
10 & +996108 & 2 & 64 & 324 & 48698 \\
20 & +998444 & 4 & 124 & 264 & 59889 \\
30 & +1059768 & 5 & 152 & 236 & 64320 \\
40 & +991634 & 7 & 207 & 181 & 69351 \\
50 & +1002523 & 9 & 254 & 134 & 66584 \\
60 & +973353 & 11 & 296 & 92 & 72352 \\
70 & +842934 & 13 & 331 & 57 & 77017 \\
80 & +811925 & 14 & 346 & 42 & 69147 \\
90 & +629754 & 16 & 372 & 16 & 76766 \\
100 & -5564631 & 18 & 388 & 0 & - \\
\hline
\end{tabular}

Table 6: Computational results of partially cyclic timetables for different levels of partiality under the 2014 demand

We plot the passenger satisfaction as a function of $\xi$ in Figure 7 . The function is rather exponential. At the partiality of $30 \%$, it outperforms the non-cyclic timetable. This is caused due to the heuristical solution approach, i.e. the non-cyclic timetable might have gotten traped in a local optimum.

More importantly, the $90 \%$ partially cyclic timetable can achieve slightly better values of the passenger satisfaction than the $30 \mathrm{~min}$ shifted cyclic timetable by having only 16 trains non-cyclic. Overall, the partially cyclic timetable achieves the passenger satisfaction values close to the ones of the non-cyclic timetable already around $60 \%$ partiality. The ratio, of the cyclic and the non-cyclic trains at this level of the partiality, is approximately $3: 1$. This means that a large improvement can be achieved by having only small adjustments made to the cyclic timetable. We further support this conclusion on the results of the hybrid cyclic timetable.

\subsubsection{Hybrid Cyclic Timetable}

The hybrid cyclic timetable as compared to the partially cyclic one, controls the ratio between the number of the cyclic and the non-cyclic trains by itself. The resulting ratio is approximately $3: 1$, 


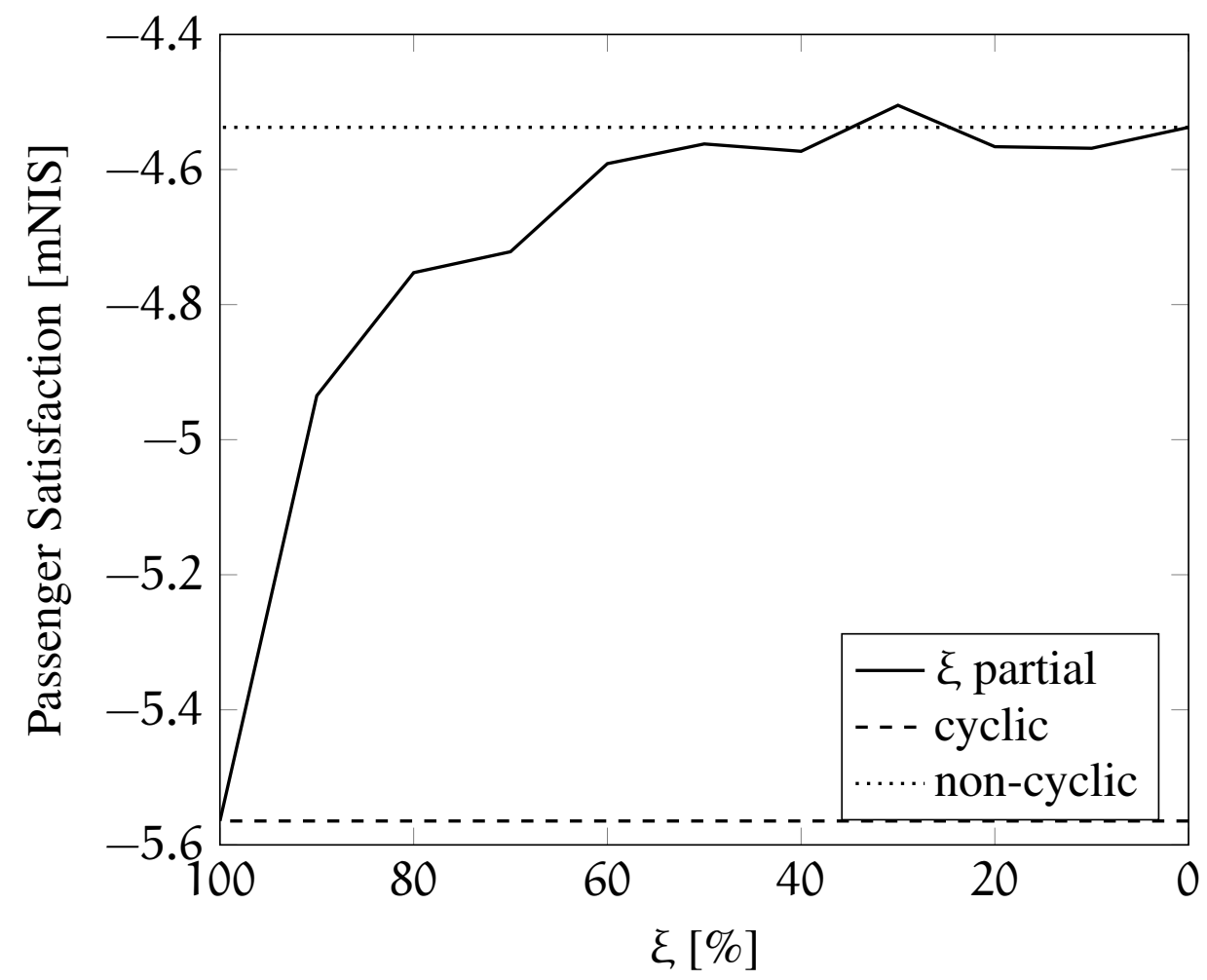

Figure 7: Passenger satisfaction of the $\xi$ partially cyclic timetable for the 2014 demand

which is the same case as for the $60 \%$ partially cyclic timetable. The passenger satisfaction is close to the one of the non-cyclic timetable (it is worse by only 13405 NIS) and of the aforementioned $60 \%$ partially cyclic timetable (it is better by 40373 NIS).

When we consider the network load (Figure 8), it is having a similar shape as the one of the noncyclic timetable. In terms of the train distribution (Table 12 in Appendix B), we can see the trend of the non-cyclic trains being mainly scheduled during the morning and evening peak hours. The solution time of the SA heuristic is 60113 seconds. Overall, the hybrid cyclic timetable has proven to be the most suitable configuration as it achieves the flexibility of the non-cyclic one, while keeping a good level of regularity.

\subsection{Summary}

Overall for this case study, the proposed hybrid timetables tend to reduce or to completely diminish the quantitative impact of the regularity (cyclicity constraints). The original gap between the flexibility of the cyclic and the non-cyclic timetable is $18.5 \%$. The $\theta$ shifted cyclic timetable is able to reduce this gap to a half (for $\theta=30$ ). The $\xi$ partially cyclic timetable can further reduce this gap to zero already at a level of $\xi=60$. The ratio of the cyclic and the non-cyclic trains under this $\xi$ is $75 \%$ and $25 \%$ respectively. This ratio of trains is further supported by the hybrid cyclic timetable, that aims at providing more even offer of the regularity to the passengers than the other hybrid timetables. Cycles 


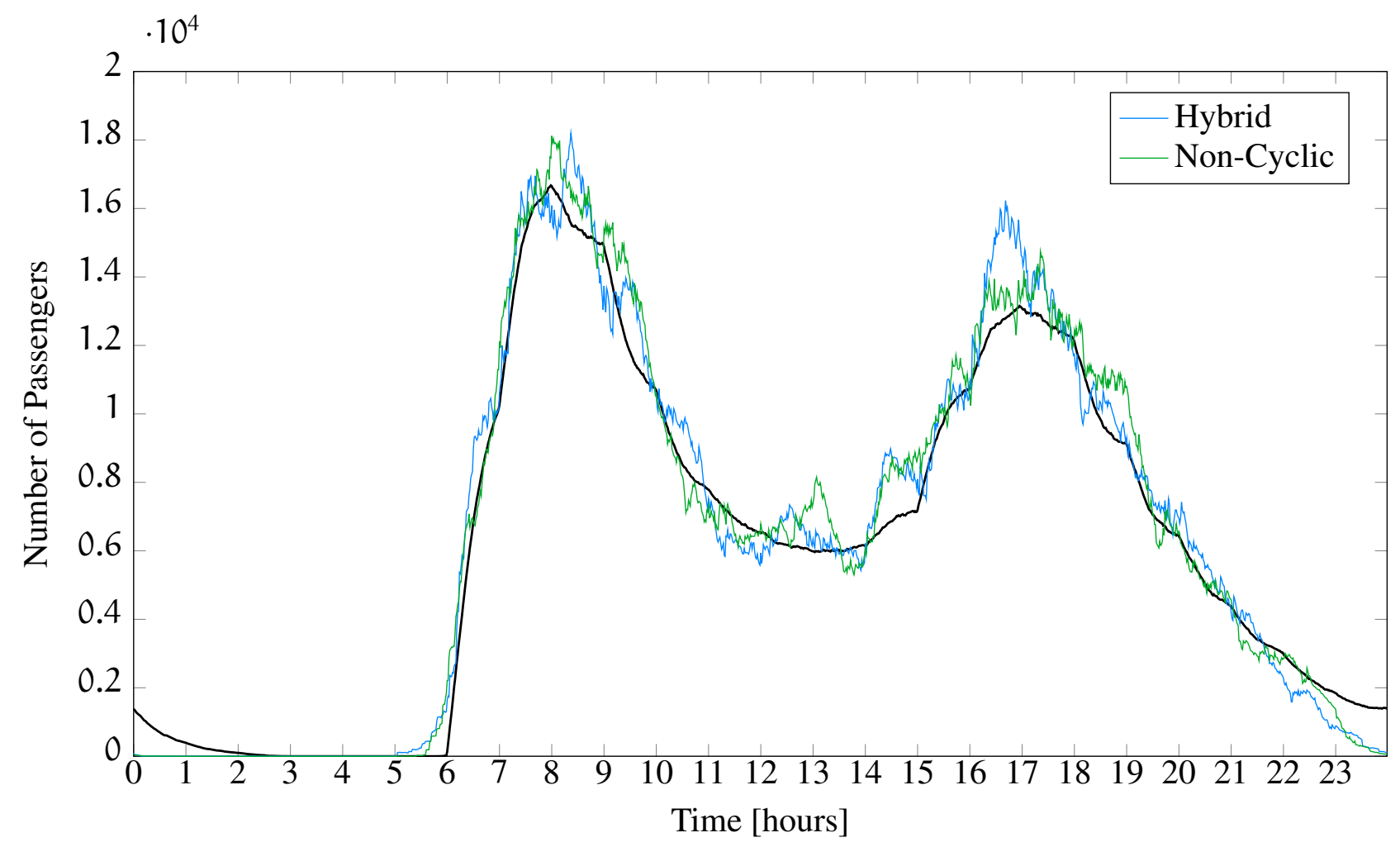

Figure 8: Network load of the non-cyclic and of the hybrid cyclic timetable for the 2014 demand

can have either only a cyclic train or both cyclic and several non-cyclic ones. The cyclic trains are used to secure the regularity and the non-cyclic trains are used to improve the service level during high demand periods.

\section{Conclusion and Future Work}

In this research, we consider the quantitative and the qualitative aspects of different passenger railway timetables. The quantitative attributes (the passenger satisfaction) show that the non-cyclic timetable is more flexible to account for the passenger demand than the cyclic one. On the other hand, the cyclic timetable has some qualitative aspects that are favored by the passengers. Namely the regularity of the service, which makes the timetable easy to be memorized by the passengers. Thus a superiority of one over the other is difficult to estimate.

In the light of these findings, we propose several combinations of the two, that would implement the qualitative aspects and allow us to explore and to reduce the quantitative impacts of the regularity. We have considered three types of hybrid timetables. The first two are generalizations of existing methods, where the relaxations of the cyclicity are explicitly modeled. The last one is an extension of the cyclic timetable imposing the non-cyclic trains to be coordinated with cyclic ones. The basic idea is that the passengers can choose between the flexibility and the regularity. We use the Passenger 
Centric Train Timetabling Problem to design the different timetables and solve it using the simulated annealing heuristic, that has proven to be an efficient instrument. We illustrate our findings on the network of Israeli Railways using the ticket selling machines' data to construct the passenger demand data. The results of our case study suggest that the hybrid cyclic timetable is the most promising approach.

Our findings have important impacts for TOCs. Indeed, cyclicity is desired in order to encourage the passengers to use train as a mode of transportation. Still, its lack of flexibility does not generate a supply configuration that is satisfying for the travelers in terms of level of service. The concept of hybrid timetables that we propose in this research allows to maintain the perception of regularity of the cyclic timetable, and to introduce the necessary flexibility for a high level of service. Although, it has not been explicitly analyzed in this paper, this added flexibility is expected to generate significant cost reductions. This will be analyzed in more detail in a future research, where a discrete choice model will be used to estimate, if a passenger would actually take the train or not. Such approach allows for a direct estimation of a TOC's profit based on the revenues and costs. Also, the actual impact of the hybrid timetables are context dependent, and other case studies should be analyzed. 


\section{Bibliography}

Brannlund, U., Lindberg, P. O., Nou, A. and Nilsson, J. E. (1998). Railway timetabling using lagrangian relaxation, Transportation Science 32(4): 358-369.

Burdett, R. and Kozan, E. (2010). A sequencing approach for creating new train timetables, $O R$ Spectrum 32(1): 163-193.

Cacchiani, V., Caprara, A. and Fischetti, M. (2012). A lagrangian heuristic for robustness, with an application to train timetabling, Transportation Science 46(1): 124-133.

Cacchiani, V., Caprara, A. and Toth, P. (2008). A column generation approach to train timetabling on a corridor, 4OR 6(2): 125-142.

Cacchiani, V., Caprara, A. and Toth, P. (2010a). Non-cyclic train timetabling and comparability graphs, Operations Research Letters 38(3): 179 - 184.

Cacchiani, V., Caprara, A. and Toth, P. (2010b). Scheduling extra freight trains on railway networks, Transportation Research Part B: Methodological 44(2): 215 - 231.

Cacchiani, V., Caprara, A. and Toth, P. (2013). Finding cliques of maximum weight on a generalization of permutation graphs, Optimization Letters 7(2): 289-296.

Caimi, G., Laumanns, M., Schüpbach, K., Wärner, S. and Fuchsberger, M. (2011). The periodic service intention as a conceptual framework for generating timetables with partial periodicity, Transportation Planning and Technology 34(4): 323-339.

Caprara, A., Fischetti, M. and Toth, P. (2002). Modeling and solving the train timetabling problem, Operations Research 50(5): 851-861.

Caprara, A., Kroon, L. G., Monaci, M., Peeters, M. and Toth, P. (2007). Passenger railway optimization, in C. Barnhart and G. Laporte (eds), Handbooks in Operations Research and Management Science, Vol. 14, Elsevier, chapter 3, pp. 129-187.

Caprara, A., Monaci, M., Toth, P. and Guida, P. L. (2006). A lagrangian heuristic algorithm for a realworld train timetabling problem, Discrete Applied Mathematics 154(5): 738 - 753. IV ALIO/EURO Workshop on Applied Combinatorial Optimization.

Carey, M. and Lockwood, D. (1995). A model, algorithms and strategy for train pathing, The Journal of the Operational Research Society 46(8): pp. 988-1005.

Cordone, R. and Redaelli, F. (2011). Optimizing the demand captured by a railway system with a regular timetable, Transportation Research Part B: Methodological 45(2): 430 - 446.

de Keizer, B., Geurs, K. and Haarsman, G. (2012). Interchanges in timetable design of railways: A closer look at customer resistance to interchange between trains., in AET (ed.), Proceedings of the European Transport Conference, Glasgow, 8-10 October 2012 (online), AET. 
Globes (2015). Israel railways passenger traffic up 7.5\% in 2014.

URL: $\quad h t t p: / / w w w . g l o b e s . c o . i l / e n / a r t i c l e-i s r a e l-r a i l w a y s-p a s s e n g e r-t r a f f i c-u p-75-i n-2014-$ 1001005774

Großmann, P., Hölldobler, S., Manthey, N., Nachtigall, K., Opitz, J. and Steinke, P. (2012). Solving periodic event scheduling problems with sat, in $\mathrm{H}$. Jiang, W. Ding, M. Ali and X. Wu (eds), Advanced Research in Applied Artificial Intelligence, Vol. 7345 of Lecture Notes in Computer Science, Springer Berlin Heidelberg, pp. 166-175.

Harrod, S. S. (2012). A tutorial on fundamental model structures for railway timetable optimization, Surveys in Operations Research and Management Science 17(2): 85 - 96.

Heydar, M., Petering, M. E. and Bergmann, D. R. (2013). Mixed integer programming for minimizing the period of a cyclic railway timetable for a single track with two train types, Computers \& Industrial Engineering 66(1): 171 - 185.

Higgins, A., Kozan, E. and Ferreira, L. (1997). Heuristic techniques for single line train scheduling, Journal of Heuristics 3(1): 43-62.

Hoppmann, H., Borndörfer, R. and Karbstein, M. (2015). Timetabling and passenger routing in public transport, Proceedings of Conference on Advanced Systems in Public Transport 2015 (CASPT2015). accepted for publication.

Jamili, A., Shafia, M., Sadjadi, S. and Tavakkoli-Moghaddam, R. (2012). Solving a periodic singletrack train timetabling problem by an efficient hybrid algorithm, Engineering Applications of Artificial Intelligence 25(4): 793 - 800. Special Section: Dependable System Modelling and Analysis.

Johnson, D., Shires, J., Nash, C. and Tyler, J. (2006). Forecasting and appraising the impact of a regular interval timetable, Transport Policy 13(5): 349 - 366.

Kaspi, M. and Raviv, T. (2013). Service-oriented line planning and timetabling for passenger trains, Transportation Science 47(3): 295-311.

Kirkpatrick, S., Gelatt, C. D. and Vecchi, M. P. (1983). Optimization by simulated annealing, SCIENCE 220(4598): 671-680.

Kroon, L. G. and Peeters, L. W. P. (2003). A variable trip time model for cyclic railway timetabling, Transportation Science 37(2): 198-212.

Liebchen, C. (2004). Symmetry for periodic railway timetables, Electronic Notes in Theoretical Computer Science 92: 34 - 51. Proceedings of \{ATMOS\} Workshop 2003.

Liebchen, C. and Peeters, L. (2009). Integral cycle bases for cyclic timetabling, Discrete Optimization 6(1): $98-109$.

Nachtigall, K. (1996). Periodic network optimization with different arc frequencies, Discrete Applied Mathematics 69: 1 - 17. 
Nachtigall, K. and Opitz, J. (2008). Solving Periodic Timetable Optimisation Problems by Modulo Simplex Calculations, in M. Fischetti and P. Widmayer (eds), 8th Workshop on Algorithmic Approaches for Transportation Modeling, Optimization, and Systems (ATMOS'08), Vol. 9 of OpenAccess Series in Informatics (OASIcs), Schloss Dagstuhl-Leibniz-Zentrum fuer Informatik, Dagstuhl, Germany.

Nachtigall, K. and Voget, S. (1996). A genetic algorithm approach to periodic railway synchronization, Computers \& Operations Research 23(5): 453 - 463.

Odijk, M. A. (1996). A constraint generation algorithm for the construction of periodic railway timetables, Transportation Research Part B: Methodological 30(6): 455 - 464.

Oliveira, E. and Smith, B. M. (2000). A job-shop scheduling model for the single-track railway scheduling problem, Technical report, University of Leeds.

Peeters, L. (2003). Cyclic Railway Timetable Optimization, ERIM Ph.D. series Research in Management, Erasmus Research inst. of Management (ERIM).

Robenek, T., Maknoon, Y., Azadeh, S. S., Chen, J. and Bierlaire, M. (2016). Passenger centric train timetabling problem, Transportation Research Part B: Methodological 89: 107 - 126.

Schmidt, M. and Schöbel, A. (2015). Timetabling with passenger routing, OR Spectrum 37(1): 75-97.

Schöbel, A. (2012). Line planning in public transportation: models and methods, OR Spectrum 34: 491-510.

Serafini, P. and Ukovich, W. (1989). A mathematical model for periodic scheduling problems, SIAM J. Discret. Math. 2(4): 550-581.

Shiftan, Y., Sharaby, N. and Solomon, C. (2008). Transport project appraisal in israel, Transportation Research Record: Journal of the Transportation Research Board (2079): 136-145.

Small, K. A. (1982). The scheduling of consumer activities: Work trips, The American Economic Review 72(3): pp. 467-479.

Vansteenwegen, P. and Oudheusden, D. V. (2006). Developing railway timetables which guarantee a better service, European Journal of Operational Research 173(1): 337 - 350.

Vansteenwegen, P. and Oudheusden, D. V. (2007). Decreasing the passenger waiting time for an intercity rail network, Transportation Research Part B: Methodological 41(4): 478 - 492.

Wardman, M. (2004). Public transport values of time, Transport Policy 11(4): 363 - 377.

Wardman, M., Shires, J., Lythgoe, W. and Tyler, J. (2004). Consumer benefits and demand impacts of regular train timetables, International Journal of Transport Management 2(1): 39 - 49. Rail Policy and Planning in Europe. 
Yang, D., Nie, L., Tan, Y., He, Z. and Zhang, Y. (2010). Working Out an Incomplete Cyclic Train Timetable for High-Speed Railways by Computer, 12th International Conference on Computer System Design and Operation in Railways and Other Transit Systems.

Zhong, J.-H., Shen, M., 0003, J. Z., Chung, H. S.-H., hui Shi, Y. and Li, Y. (2013). A differential evolution algorithm with dual populations for solving periodic railway timetable scheduling problem., IEEE Trans. Evolutionary Computation 17(4): 512-527.

\section{A Data Description}

The data used in this study were taken obtained from the IR's website (www.rail.co.il/EN) and from other studies concerning the IR's network (Kaspi and Raviv (2013)). An algorithm in Java has been coded, in order to find a set of all possible paths between every OD pair. The algorithm allows a maximum of 3 consecutive lines to get from an origin to a destination. The algorithm iterates through all OD pairs, where at first it considers the paths that consist of a single line and then the paths that would transfer from the currently selected line up to two other lines. The transfer from one line to another can be made only at one of the designated transfer points (there are 7 recommended interchange stations in the network of Israel - Figure 1). Note that the fact, that a transfer is actually possible depends on the operated timetable. Therefore, some paths might be eliminated later on by the PCTTP model itself. When all the possible paths are generated, the algorithm removes the paths that a passenger would not consider (note that these rules are related to the network layout of Israel and might differ for other case studies):

- paths that consist of several lines including a direct line between the given OD pair, where both options travel on the same infrastructure (i.e. the passenger would rather stay on the direct line instead of transferring to another line).

- paths that consist of several lines, where two of them can reach the given destination. Changing one train to another, when both of them are going to the same destination would not make sense (the same does not happen for the origin).

- paths that take $25 \%$ longer generalized time (sum of in-vehicle-times and transfer penalties) than the shortest possible path.

- paths that consist of redundant transfers, i.e. transferring from one line to another line that covers the same stations.

Each passenger is having one extra path that represents their shortest path between an origin and their destination, when using the first scheduled train after the planning horizon. This path represents a penalty of not being served. The largest number of paths between an origin and a destination is 73 and the total amount of paths in the network is 21469.

The traveling times have been extracted from the IR's website along with the dwell times at stations that remain fixed (as of the timetable 2013/14). The minimum transfer time has been set to 
4 minutes as in Kaspi and Raviv (2013). The Value Of Time of commuters in Israel as of the year 2012 is 21.12 New Israeli Shekel (NIS)/hour (the updated value was given to us by the author of Shiftan et al. (2008)). The $\beta$ parameters and their values are as follows: $\beta_{W}=2.5$ (Wardman (2004)), $\beta_{\mathrm{T}}=10$ (de Keizer et al. (2012)), $\beta_{\mathrm{E}}=0.5$ and $\beta_{\mathrm{L}}=1$ (Small (1982)).

\section{A.1 Passenger}

The OD flows were kindly provided by Mor Kaspi and Tal Raviv, who have cleaned the ticket selling machines' data for the year 2008 and produced the flows of an average working day in Israel. They have used this data in their study Kaspi and Raviv (2013). The OD matrix consists of hourly passenger rates between 6 a.m. and 1 a.m. The flows were smoothed into minutes by using non-homogenous Poisson process, where the hourly flows per OD pair were used as the arrival rate variable. Since the schedule passenger delay is related to the destination, we have added the time that it takes to get from an origin to a destination using the shortest path (if the path consisted of transfers, we assumed the perfect connection, i.e. only the minimum transfer time without any additional waiting at the transfer station). In total there are 1505 out of 2162 OD pairs with 126036 passengers.

\section{A.2 Operator}

As no information about the rolling stock fleet of IR is available, we have introduced the following assumptions:

- The fleet is homogenous

- A train unit has a passenger capacity of 250

- Each train can consist of up to 2 train units

- The number of train units remains the same between the start and the end station of a train

In order to verify that the assumed train capacity is reasonable, we have solved the un-capacitated PCTTP of the IR 13/14 timetable under the 2008 demand, where the average train occupation was 172 passengers per train per segment (pptps), minimum occupation was 0 pptps, maximum occupation 1188 pptps and median was 124 pptps. Thus the capacity of 250 passengers per train unit offers a good level of service.

\section{B Train Distributions}

In the tables of this section, we present the train distributions of various types of timetables. The first row of each table, represents the hour of the day. Each subsequent row, represents each of the lines in the network (in total 34 lines). The number in each cell gives the information about the number of trains scheduled in that given hour on that given line. The minimum value found is 0 and the maximum value found is 4 . 


\begin{tabular}{|c|c|c|c|c|c|c|c|c|c|c|c|c|c|c|c|c|c|c|c|c|c|c|c|}
\hline 0 & 1 & 2 & 3 & 4 & 5 & 6 & 7 & 8 & 9 & 10 & 11 & 12 & 13 & 14 & 15 & 16 & 17 & 18 & 19 & 20 & 21 & 22 & 23 \\
\hline 0 & 0 & 0 & 0 & 1 & 1 & 1 & 1 & 1 & 1 & 1 & 1 & 1 & 1 & 1 & 1 & 1 & 1 & 1 & 1 & 1 & 1 & 0 & 0 \\
\hline 0 & 0 & 0 & 0 & 0 & 1 & 1 & 1 & 1 & 1 & 1 & 1 & 1 & 1 & 1 & 1 & 1 & 1 & 1 & 1 & 1 & 1 & 1 & 0 \\
\hline 0 & 0 & 0 & 0 & 0 & 0 & 0 & 0 & 0 & 0 & 0 & 0 & 0 & 0 & 0 & 0 & 0 & 1 & 0 & 0 & 0 & 0 & 0 & 0 \\
\hline 0 & 0 & 0 & 0 & 0 & 0 & 1 & 0 & 0 & 0 & 0 & 0 & 0 & 0 & 0 & 0 & 0 & 0 & 0 & 0 & 0 & 0 & 0 & 0 \\
\hline 0 & 0 & 0 & 0 & 0 & 1 & 1 & 1 & 1 & 1 & 1 & 1 & 1 & 1 & 1 & 1 & 1 & 1 & 1 & 1 & 1 & 0 & 0 & 0 \\
\hline 0 & 0 & 0 & 0 & 0 & 0 & 1 & 1 & 1 & 1 & 1 & 1 & 1 & 1 & 1 & 1 & 1 & 1 & 1 & 1 & 1 & 1 & 1 & 0 \\
\hline 0 & 0 & 0 & 0 & 0 & 1 & 1 & 1 & 1 & 1 & 0 & 0 & 0 & 1 & 1 & 1 & 1 & 1 & 1 & 0 & 0 & 0 & 0 & 0 \\
\hline 0 & 0 & 0 & 0 & 0 & 0 & 1 & 1 & 1 & 1 & 1 & 0 & 0 & 0 & 1 & 1 & 1 & 1 & 1 & 1 & 0 & 0 & 0 & 0 \\
\hline 0 & 0 & 0 & 0 & 0 & 0 & 1 & 1 & 1 & 1 & 0 & 0 & 0 & 1 & 1 & 1 & 1 & 1 & 1 & 1 & 1 & 1 & 0 & 0 \\
\hline 0 & 0 & 0 & 0 & 0 & 1 & 1 & 1 & 1 & 1 & 1 & 0 & 0 & 0 & 1 & 1 & 1 & 1 & 1 & 1 & 0 & 0 & 0 & 0 \\
\hline 0 & 0 & 0 & 0 & 0 & 0 & 1 & 1 & 1 & 1 & 1 & 1 & 1 & 1 & 1 & 1 & 1 & 1 & 1 & 1 & 1 & 0 & 0 & 0 \\
\hline 0 & 0 & 0 & 0 & 0 & 0 & 1 & 1 & 1 & 1 & 1 & 1 & 1 & 1 & 1 & 1 & 1 & 1 & 1 & 1 & 1 & 1 & 0 & 0 \\
\hline 0 & 0 & 0 & 0 & 0 & 1 & 1 & 1 & 1 & 1 & 1 & 1 & 1 & 1 & 1 & 1 & 1 & 1 & 1 & 1 & 1 & 1 & 1 & 0 \\
\hline 0 & 0 & 0 & 0 & 0 & 1 & 1 & 1 & 1 & 1 & 1 & 1 & 1 & 1 & 1 & 1 & 1 & 1 & 1 & 1 & 1 & 1 & 0 & 0 \\
\hline 0 & 0 & 0 & 0 & 0 & 1 & 0 & 0 & 1 & 0 & 0 & 0 & 0 & 0 & 0 & 0 & 1 & 0 & 0 & 1 & 0 & 0 & 0 & 0 \\
\hline 0 & 0 & 0 & 0 & 0 & 0 & 0 & 1 & 0 & 0 & 0 & 1 & 0 & 0 & 0 & 0 & 0 & 1 & 0 & 0 & 1 & 0 & 0 & 0 \\
\hline 0 & 0 & 0 & 0 & 0 & 0 & 1 & 1 & 1 & 0 & 0 & 0 & 0 & 0 & 0 & 1 & 1 & 1 & 1 & 0 & 0 & 0 & 0 & 0 \\
\hline 0 & 0 & 0 & 0 & 0 & 0 & 0 & 1 & 1 & 1 & 0 & 0 & 0 & 0 & 0 & 1 & 1 & 1 & 1 & 0 & 0 & 0 & 0 & 0 \\
\hline 0 & 0 & 0 & 0 & 0 & 1 & 1 & 1 & 1 & 1 & 1 & 1 & 1 & 1 & 1 & 1 & 1 & 1 & 1 & 1 & 1 & 1 & 1 & 0 \\
\hline 0 & 0 & 0 & 0 & 0 & 1 & 1 & 1 & 1 & 1 & 1 & 1 & 1 & 1 & 1 & 1 & 1 & 1 & 1 & 1 & 1 & 1 & 1 & 0 \\
\hline 0 & 0 & 0 & 0 & 0 & 0 & 1 & 1 & 1 & 1 & 1 & 1 & 1 & 1 & 1 & 1 & 1 & 1 & 1 & 1 & 1 & 0 & 0 & 0 \\
\hline 0 & 0 & 0 & 0 & 0 & 0 & 1 & 1 & 1 & 1 & 1 & 1 & 1 & 1 & 1 & 1 & 1 & 1 & 1 & 1 & 1 & 1 & 0 & 0 \\
\hline 0 & 0 & 0 & 0 & 0 & 1 & 1 & 1 & 1 & 0 & 0 & 0 & 0 & 0 & 1 & 1 & 1 & 1 & 1 & 0 & 0 & 0 & 0 & 0 \\
\hline 0 & 0 & 0 & 0 & 0 & 0 & 1 & 1 & 1 & 0 & 0 & 0 & 0 & 0 & 0 & 1 & 1 & 1 & 1 & 1 & 0 & 0 & 0 & 0 \\
\hline 0 & 0 & 0 & 0 & 0 & 1 & 1 & 1 & 1 & 1 & 1 & 1 & 1 & 1 & 1 & 1 & 1 & 1 & 1 & 1 & 1 & 0 & 1 & 1 \\
\hline 0 & 0 & 0 & 0 & 0 & 1 & 1 & 1 & 1 & 1 & 1 & 1 & 1 & 1 & 1 & 1 & 1 & 1 & 1 & 1 & 1 & 1 & 1 & 0 \\
\hline 0 & 0 & 0 & 0 & 0 & 0 & 1 & 1 & 1 & 1 & 0 & 1 & 1 & 1 & 1 & 1 & 1 & 1 & 1 & 1 & 0 & 0 & 0 & 0 \\
\hline 0 & 0 & 0 & 0 & 0 & 0 & 1 & 1 & 1 & 1 & 0 & 1 & 1 & 1 & 1 & 1 & 1 & 1 & 1 & 1 & 1 & 0 & 0 & 0 \\
\hline 0 & 0 & 0 & 0 & 0 & 0 & 0 & 1 & 0 & 0 & 0 & 0 & 0 & 0 & 0 & 0 & 0 & 0 & 0 & 0 & 0 & 0 & 0 & 0 \\
\hline 0 & 0 & 0 & 0 & 0 & 0 & 0 & 0 & 0 & 0 & 0 & 0 & 0 & 0 & 0 & 0 & 1 & 0 & 0 & 0 & 0 & 0 & 0 & 0 \\
\hline 0 & 0 & 0 & 0 & 0 & 0 & 0 & 1 & 1 & 0 & 0 & 0 & 0 & 0 & 0 & 1 & 1 & 1 & 1 & 0 & 0 & 0 & 0 & 0 \\
\hline 0 & 0 & 0 & 0 & 0 & 0 & 1 & 1 & 1 & 1 & 0 & 0 & 0 & 0 & 0 & 0 & 1 & 1 & 1 & 0 & 0 & 0 & 0 & 0 \\
\hline 0 & 0 & 0 & 0 & 0 & 0 & 1 & 1 & 1 & 1 & 0 & 1 & 0 & 0 & 1 & 0 & 1 & 1 & 1 & 0 & 0 & 0 & 0 & 0 \\
\hline 0 & 0 & 0 & 0 & 0 & 0 & 1 & 1 & 1 & 1 & 1 & 0 & 1 & 0 & 1 & 1 & 1 & 1 & 1 & 0 & 0 & 0 & 0 & 0 \\
\hline
\end{tabular}

Table 7: Train distribution of the IR 13/14 timetable 


\begin{tabular}{|c|c|c|c|c|c|c|c|c|c|c|c|c|c|c|c|c|c|c|c|c|c|c|c|}
\hline 0 & 1 & 2 & 3 & 4 & 5 & 6 & 7 & 8 & 9 & 10 & 11 & 12 & 13 & 14 & 15 & 16 & 17 & 18 & 19 & 20 & 21 & 22 & 23 \\
\hline 0 & 0 & 0 & 0 & 1 & 1 & 1 & 1 & 1 & 1 & 1 & 1 & 1 & 1 & 1 & 1 & 1 & 1 & 1 & 1 & 1 & 1 & 0 & 0 \\
\hline 0 & 0 & 0 & 0 & 0 & 1 & 1 & 1 & 1 & 1 & 1 & 1 & 1 & 1 & 1 & 1 & 1 & 1 & 1 & 1 & 1 & 1 & 1 & 0 \\
\hline 0 & 0 & 0 & 0 & 0 & 0 & 0 & 0 & 0 & 0 & 0 & 0 & 0 & 0 & 0 & 0 & 0 & 1 & 0 & 0 & 0 & 0 & 0 & 0 \\
\hline 0 & 0 & 0 & 0 & 0 & 0 & 1 & 0 & 0 & 0 & 0 & 0 & 0 & 0 & 0 & 0 & 0 & 0 & 0 & 0 & 0 & 0 & 0 & 0 \\
\hline 0 & 0 & 0 & 0 & 0 & 1 & 1 & 1 & 1 & 1 & 1 & 1 & 1 & 1 & 1 & 1 & 1 & 1 & 1 & 1 & 1 & 0 & 0 & 0 \\
\hline 0 & 0 & 0 & 0 & 0 & 0 & 1 & 1 & 1 & 1 & 1 & 1 & 1 & 1 & 1 & 1 & 1 & 1 & 1 & 1 & 1 & 1 & 1 & 0 \\
\hline 0 & 0 & 0 & 0 & 0 & 1 & 1 & 1 & 1 & 1 & 1 & 1 & 1 & 1 & 1 & 1 & 1 & 1 & 1 & 0 & 0 & 0 & 0 & 0 \\
\hline 0 & 0 & 0 & 0 & 0 & 0 & 1 & 1 & 1 & 1 & 1 & 1 & 1 & 1 & 1 & 1 & 1 & 1 & 1 & 1 & 0 & 0 & 0 & 0 \\
\hline 0 & 0 & 0 & 0 & 0 & 0 & 1 & 1 & 1 & 1 & 1 & 1 & 1 & 1 & 1 & 1 & 1 & 1 & 1 & 1 & 1 & 1 & 0 & 0 \\
\hline 0 & 0 & 0 & 0 & 0 & 1 & 1 & 1 & 1 & 1 & 1 & 1 & 1 & 1 & 1 & 1 & 1 & 1 & 1 & 1 & 0 & 0 & 0 & 0 \\
\hline 0 & 0 & 0 & 0 & 0 & 0 & 1 & 1 & 1 & 1 & 1 & 1 & 1 & 1 & 1 & 1 & 1 & 1 & 1 & 1 & 1 & 0 & 0 & 0 \\
\hline 0 & 0 & 0 & 0 & 0 & 0 & 1 & 1 & 1 & 1 & 1 & 1 & 1 & 1 & 1 & 1 & 1 & 1 & 1 & 1 & 1 & 1 & 0 & 0 \\
\hline 0 & 0 & 0 & 0 & 0 & 1 & 1 & 1 & 1 & 1 & 1 & 1 & 1 & 1 & 1 & 1 & 1 & 1 & 1 & 1 & 1 & 1 & 1 & 0 \\
\hline 0 & 0 & 0 & 0 & 0 & 1 & 1 & 1 & 1 & 1 & 1 & 1 & 1 & 1 & 1 & 1 & 1 & 1 & 1 & 1 & 1 & 1 & 0 & 0 \\
\hline 0 & 0 & 0 & 0 & 0 & 1 & 1 & 1 & 1 & 1 & 1 & 1 & 1 & 1 & 1 & 1 & 1 & 1 & 1 & 1 & 0 & 0 & 0 & 0 \\
\hline 0 & 0 & 0 & 0 & 0 & 0 & 0 & 1 & 1 & 1 & 1 & 1 & 1 & 1 & 1 & 1 & 1 & 1 & 1 & 1 & 1 & 0 & 0 & 0 \\
\hline 0 & 0 & 0 & 0 & 0 & 0 & 1 & 1 & 1 & 1 & 1 & 1 & 1 & 1 & 1 & 1 & 1 & 1 & 1 & 0 & 0 & 0 & 0 & 0 \\
\hline 0 & 0 & 0 & 0 & 0 & 0 & 0 & 1 & 1 & 1 & 1 & 1 & 1 & 1 & 1 & 1 & 1 & 1 & 1 & 0 & 0 & 0 & 0 & 0 \\
\hline 0 & 0 & 0 & 0 & 0 & 1 & 1 & 1 & 1 & 1 & 1 & 1 & 1 & 1 & 1 & 1 & 1 & 1 & 1 & 1 & 1 & 1 & 1 & 0 \\
\hline 0 & 0 & 0 & 0 & 0 & 1 & 1 & 1 & 1 & 1 & 1 & 1 & 1 & 1 & 1 & 1 & 1 & 1 & 1 & 1 & 1 & 1 & 1 & 0 \\
\hline 0 & 0 & 0 & 0 & 0 & 0 & 1 & 1 & 1 & 1 & 1 & 1 & 1 & 1 & 1 & 1 & 1 & 1 & 1 & 1 & 1 & 0 & 0 & 0 \\
\hline 0 & 0 & 0 & 0 & 0 & 0 & 1 & 1 & 1 & 1 & 1 & 1 & 1 & 1 & 1 & 1 & 1 & 1 & 1 & 1 & 1 & 1 & 0 & 0 \\
\hline 0 & 0 & 0 & 0 & 0 & 1 & 1 & 1 & 1 & 1 & 1 & 1 & 1 & 1 & 1 & 1 & 1 & 1 & 1 & 0 & 0 & 0 & 0 & 0 \\
\hline 0 & 0 & 0 & 0 & 0 & 0 & 1 & 1 & 1 & 1 & 1 & 1 & 1 & 1 & 1 & 1 & 1 & 1 & 1 & 1 & 0 & 0 & 0 & 0 \\
\hline 0 & 0 & 0 & 0 & 0 & 1 & 1 & 1 & 1 & 1 & 1 & 1 & 1 & 1 & 1 & 1 & 1 & 1 & 1 & 1 & 1 & 1 & 1 & 1 \\
\hline 0 & 0 & 0 & 0 & 0 & 1 & 1 & 1 & 1 & 1 & 1 & 1 & 1 & 1 & 1 & 1 & 1 & 1 & 1 & 1 & 1 & 1 & 1 & 0 \\
\hline 0 & 0 & 0 & 0 & 0 & 0 & 1 & 1 & 1 & 1 & 1 & 1 & 1 & 1 & 1 & 1 & 1 & 1 & 1 & 1 & 0 & 0 & 0 & 0 \\
\hline 0 & 0 & 0 & 0 & 0 & 0 & 1 & 1 & 1 & 1 & 1 & 1 & 1 & 1 & 1 & 1 & 1 & 1 & 1 & 1 & 1 & 0 & 0 & 0 \\
\hline 0 & 0 & 0 & 0 & 0 & 0 & 0 & 1 & 0 & 0 & 0 & 0 & 0 & 0 & 0 & 0 & 0 & 0 & 0 & 0 & 0 & 0 & 0 & 0 \\
\hline 0 & 0 & 0 & 0 & 0 & 0 & 0 & 0 & 0 & 0 & 0 & 0 & 0 & 0 & 0 & 0 & 1 & 0 & 0 & 0 & 0 & 0 & 0 & 0 \\
\hline 0 & 0 & 0 & 0 & 0 & 0 & 0 & 1 & 1 & 1 & 1 & 1 & 1 & 1 & 1 & 1 & 1 & 1 & 1 & 0 & 0 & 0 & 0 & 0 \\
\hline 0 & 0 & 0 & 0 & 0 & 0 & 1 & 1 & 1 & 1 & 1 & 1 & 1 & 1 & 1 & 1 & 1 & 1 & 1 & 0 & 0 & 0 & 0 & 0 \\
\hline 0 & 0 & 0 & 0 & 0 & 0 & 1 & 1 & 1 & 1 & 1 & 1 & 1 & 1 & 1 & 1 & 1 & 1 & 1 & 0 & 0 & 0 & 0 & 0 \\
\hline 0 & 0 & 0 & 0 & 0 & 0 & 1 & 1 & 1 & 1 & 1 & 1 & 1 & 1 & 1 & 1 & 1 & 1 & 1 & 0 & 0 & 0 & 0 & 0 \\
\hline
\end{tabular}

Table 8: Train distribution of the IR $13 / 14$ as strictly cyclic timetable 


\begin{tabular}{|c|c|c|c|c|c|c|c|c|c|c|c|c|c|c|c|c|c|c|c|c|c|c|c|}
\hline 0 & 1 & 2 & 3 & 4 & 5 & 6 & 7 & 8 & 9 & 10 & 11 & 12 & 13 & 14 & 15 & 16 & 17 & 18 & 19 & 20 & 21 & 22 & 23 \\
\hline 0 & 0 & 0 & 0 & 1 & 1 & 1 & 1 & 1 & 1 & 1 & 1 & 0 & 1 & 0 & 1 & 1 & 1 & 1 & 1 & 0 & 1 & 1 & 0 \\
\hline 0 & 0 & 0 & 0 & 0 & 0 & 1 & 1 & 1 & 1 & 1 & 1 & 1 & 1 & 1 & 1 & 1 & 1 & 1 & 1 & 1 & 1 & 1 & 1 \\
\hline 0 & 0 & 0 & 0 & 0 & 0 & 1 & 0 & 0 & 0 & 0 & 0 & 0 & 0 & 0 & 0 & 0 & 0 & 0 & 0 & 0 & 0 & 0 & 0 \\
\hline 0 & 0 & 0 & 0 & 0 & 0 & 0 & 0 & 0 & 0 & 0 & 0 & 0 & 0 & 0 & 0 & 1 & 0 & 0 & 0 & 0 & 0 & 0 & 0 \\
\hline 0 & 0 & 0 & 1 & 0 & 1 & 1 & 1 & 1 & 1 & 1 & 1 & 1 & 1 & 1 & 1 & 1 & 1 & 0 & 1 & 1 & 0 & 0 & 0 \\
\hline 0 & 0 & 0 & 0 & 0 & 0 & 1 & 1 & 1 & 1 & 1 & 1 & 1 & 1 & 1 & 1 & 1 & 1 & 1 & 1 & 1 & 1 & 1 & 0 \\
\hline 0 & 0 & 0 & 0 & 0 & 0 & 1 & 1 & 1 & 1 & 0 & 1 & 0 & 0 & 1 & 1 & 1 & 1 & 1 & 1 & 0 & 0 & 0 & 0 \\
\hline 0 & 0 & 0 & 0 & 0 & 0 & 1 & 1 & 1 & 1 & 1 & 1 & 0 & 1 & 0 & 0 & 1 & 1 & 1 & 1 & 0 & 0 & 0 & 0 \\
\hline 0 & 0 & 0 & 0 & 0 & 0 & 1 & 1 & 1 & 1 & 1 & 0 & 0 & 0 & 1 & 1 & 1 & 1 & 1 & 1 & 1 & 1 & 0 & 0 \\
\hline 0 & 0 & 0 & 0 & 0 & 1 & 1 & 1 & 1 & 1 & 1 & 1 & 1 & 0 & 1 & 1 & 1 & 1 & 0 & 0 & 0 & 0 & 0 & 0 \\
\hline 0 & 0 & 0 & 0 & 0 & 1 & 1 & 1 & 1 & 1 & 1 & 1 & 1 & 0 & 1 & 1 & 1 & 1 & 1 & 0 & 1 & 1 & 0 & 0 \\
\hline 0 & 0 & 0 & 0 & 0 & 1 & 1 & 1 & 1 & 0 & 0 & 1 & 0 & 1 & 1 & 1 & 1 & 1 & 1 & 1 & 1 & 1 & 1 & 0 \\
\hline 0 & 0 & 0 & 0 & 0 & 1 & 1 & 1 & 1 & 1 & 1 & 1 & 1 & 1 & 1 & 1 & 1 & 1 & 1 & 1 & 1 & 1 & 1 & 0 \\
\hline 0 & 0 & 0 & 0 & 1 & 1 & 1 & 1 & 1 & 1 & 1 & 1 & 1 & 1 & 1 & 1 & 1 & 1 & 1 & 1 & 1 & 0 & 0 & 0 \\
\hline 0 & 0 & 0 & 0 & 0 & 0 & 0 & 1 & 0 & 0 & 0 & 0 & 0 & 0 & 0 & 0 & 0 & 1 & 0 & 0 & 0 & 0 & 0 & 0 \\
\hline 0 & 0 & 0 & 0 & 0 & 0 & 0 & 1 & 0 & 0 & 0 & 0 & 0 & 0 & 0 & 0 & 0 & 1 & 0 & 0 & 0 & 0 & 0 & 0 \\
\hline 0 & 0 & 0 & 0 & 0 & 1 & 1 & 1 & 1 & 0 & 1 & 0 & 0 & 0 & 0 & 1 & 0 & 1 & 0 & 0 & 0 & 0 & 0 & 0 \\
\hline 0 & 0 & 0 & 0 & 0 & 0 & 0 & 1 & 0 & 1 & 0 & 1 & 0 & 1 & 0 & 0 & 1 & 1 & 0 & 0 & 1 & 0 & 0 & 0 \\
\hline 0 & 0 & 0 & 0 & 0 & 0 & 1 & 1 & 1 & 1 & 1 & 1 & 1 & 1 & 1 & 1 & 1 & 1 & 1 & 1 & 1 & 1 & 1 & 1 \\
\hline 1 & 0 & 0 & 0 & 0 & 0 & 1 & 1 & 1 & 1 & 1 & 1 & 1 & 1 & 1 & 1 & 1 & 1 & 1 & 1 & 1 & 1 & 0 & 0 \\
\hline 0 & 0 & 0 & 0 & 0 & 0 & 0 & 1 & 1 & 1 & 1 & 1 & 1 & 1 & 1 & 1 & 1 & 1 & 1 & 0 & 1 & 1 & 0 & 0 \\
\hline 0 & 0 & 0 & 0 & 0 & 1 & 1 & 1 & 1 & 1 & 1 & 1 & 1 & 0 & 1 & 1 & 1 & 1 & 1 & 1 & 1 & 0 & 1 & 0 \\
\hline 0 & 0 & 0 & 0 & 0 & 0 & 1 & 0 & 1 & 0 & 0 & 0 & 0 & 1 & 0 & 1 & 1 & 1 & 1 & 1 & 1 & 0 & 0 & 0 \\
\hline 0 & 0 & 0 & 0 & 0 & 1 & 1 & 1 & 1 & 1 & 0 & 0 & 0 & 0 & 1 & 0 & 1 & 1 & 0 & 0 & 0 & 0 & 0 & 0 \\
\hline 0 & 0 & 0 & 0 & 0 & 1 & 1 & 1 & 1 & 1 & 1 & 1 & 1 & 1 & 1 & 1 & 1 & 1 & 1 & 1 & 1 & 1 & 1 & 0 \\
\hline 0 & 0 & 0 & 0 & 0 & 1 & 1 & 1 & 1 & 1 & 1 & 1 & 1 & 1 & 1 & 1 & 1 & 1 & 1 & 1 & 1 & 1 & 1 & 0 \\
\hline 0 & 0 & 0 & 0 & 0 & 0 & 1 & 1 & 1 & 1 & 1 & 1 & 1 & 1 & 1 & 1 & 1 & 1 & 0 & 1 & 0 & 0 & 0 & 0 \\
\hline 0 & 0 & 0 & 0 & 0 & 0 & 1 & 1 & 1 & 1 & 1 & 1 & 1 & 1 & 1 & 1 & 1 & 0 & 1 & 1 & 1 & 0 & 0 & 0 \\
\hline 0 & 0 & 0 & 0 & 0 & 0 & 0 & 0 & 0 & 0 & 0 & 0 & 0 & 0 & 0 & 0 & 0 & 1 & 0 & 0 & 0 & 0 & 0 & 0 \\
\hline 0 & 0 & 0 & 0 & 0 & 0 & 1 & 0 & 0 & 0 & 0 & 0 & 0 & 0 & 0 & 0 & 0 & 0 & 0 & 0 & 0 & 0 & 0 & 0 \\
\hline 0 & 0 & 0 & 0 & 0 & 0 & 0 & 1 & 1 & 0 & 0 & 0 & 1 & 1 & 0 & 0 & 1 & 0 & 0 & 1 & 0 & 0 & 0 & 0 \\
\hline 0 & 0 & 0 & 0 & 0 & 0 & 1 & 1 & 1 & 1 & 1 & 0 & 1 & 0 & 0 & 0 & 0 & 0 & 0 & 0 & 0 & 0 & 0 & 0 \\
\hline 0 & 0 & 0 & 0 & 0 & 0 & 1 & 0 & 0 & 0 & 0 & 1 & 1 & 1 & 1 & 1 & 1 & 1 & 1 & 0 & 0 & 0 & 0 & 0 \\
\hline 0 & 0 & 0 & 0 & 0 & 0 & 1 & 1 & 1 & 1 & 0 & 1 & 0 & 1 & 0 & 1 & 1 & 1 & 1 & 0 & 0 & 0 & 0 & 1 \\
\hline
\end{tabular}

Table 9: Train distribution of the cyclic timetable under the 2014 demand 


\begin{tabular}{|c|c|c|c|c|c|c|c|c|c|c|c|c|c|c|c|c|c|c|c|c|c|c|c|}
\hline 0 & 1 & 2 & 3 & 4 & 5 & 6 & 7 & 8 & 9 & 10 & 11 & 12 & 13 & 14 & 15 & 16 & 17 & 18 & 19 & 20 & 21 & 22 & 23 \\
\hline 0 & 0 & 0 & 0 & 0 & 2 & 2 & 2 & 1 & 1 & 1 & 1 & 0 & 1 & 1 & 1 & 1 & 1 & 1 & 1 & 0 & 1 & 0 & 0 \\
\hline 0 & 0 & 0 & 0 & 0 & 0 & 1 & 1 & 1 & 1 & 1 & 0 & 1 & 0 & 1 & 2 & 2 & 2 & 2 & 0 & 2 & 0 & 0 & 1 \\
\hline 0 & 0 & 0 & 0 & 0 & 0 & 1 & 0 & 0 & 0 & 0 & 0 & 0 & 0 & 0 & 0 & 0 & 0 & 0 & 0 & 0 & 0 & 0 & 0 \\
\hline 0 & 0 & 0 & 0 & 0 & 0 & 0 & 0 & 0 & 0 & 1 & 0 & 0 & 0 & 0 & 0 & 0 & 0 & 0 & 0 & 0 & 0 & 0 & 0 \\
\hline 0 & 0 & 0 & 0 & 0 & 0 & 2 & 4 & 1 & 1 & 0 & 1 & 0 & 1 & 1 & 1 & 0 & 2 & 0 & 1 & 0 & 1 & 0 & 0 \\
\hline 0 & 0 & 0 & 0 & 0 & 0 & 2 & 1 & 2 & 1 & 1 & 1 & 1 & 0 & 2 & 2 & 1 & 1 & 1 & 1 & 0 & 0 & 0 & 0 \\
\hline 0 & 0 & 0 & 0 & 0 & 1 & 1 & 1 & 0 & 1 & 1 & 0 & 0 & 0 & 1 & 0 & 3 & 1 & 1 & 0 & 0 & 0 & 0 & 0 \\
\hline 0 & 0 & 0 & 0 & 0 & 0 & 1 & 2 & 2 & 2 & 0 & 0 & 0 & 0 & 0 & 1 & 2 & 0 & 0 & 0 & 0 & 1 & 0 & 0 \\
\hline 0 & 0 & 0 & 0 & 0 & 0 & 0 & 1 & 2 & 0 & 0 & 0 & 1 & 0 & 1 & 2 & 2 & 1 & 1 & 1 & 0 & 1 & 0 & 0 \\
\hline 0 & 0 & 0 & 0 & 0 & 0 & 2 & 2 & 1 & 1 & 0 & 0 & 0 & 0 & 1 & 1 & 1 & 1 & 1 & 0 & 1 & 0 & 0 & 0 \\
\hline 0 & 0 & 0 & 0 & 0 & 1 & 1 & 2 & 1 & 1 & 1 & 0 & 1 & 1 & 1 & 1 & 1 & 1 & 0 & 1 & 0 & 1 & 0 & 0 \\
\hline 0 & 0 & 0 & 0 & 0 & 0 & 1 & 1 & 1 & 0 & 1 & 0 & 1 & 1 & 1 & 2 & 2 & 2 & 1 & 0 & 1 & 0 & 1 & 0 \\
\hline 0 & 0 & 0 & 0 & 0 & 1 & 1 & 2 & 1 & 1 & 1 & 1 & 1 & 0 & 2 & 1 & 3 & 1 & 1 & 1 & 0 & 0 & 0 & 0 \\
\hline 0 & 0 & 0 & 0 & 0 & 2 & 2 & 2 & 2 & 0 & 1 & 0 & 1 & 2 & 0 & 1 & 1 & 0 & 2 & 0 & 1 & 0 & 0 & 0 \\
\hline 0 & 0 & 0 & 0 & 0 & 0 & 0 & 1 & 1 & 0 & 0 & 0 & 0 & 0 & 0 & 0 & 1 & 0 & 1 & 0 & 0 & 0 & 0 & 0 \\
\hline 0 & 0 & 0 & 0 & 0 & 0 & 1 & 0 & 1 & 0 & 0 & 0 & 0 & 0 & 0 & 0 & 1 & 0 & 1 & 0 & 0 & 0 & 0 & 0 \\
\hline 0 & 0 & 0 & 0 & 0 & 0 & 1 & 1 & 1 & 1 & 0 & 1 & 0 & 0 & 1 & 0 & 1 & 0 & 0 & 0 & 0 & 0 & 0 & 0 \\
\hline 0 & 0 & 0 & 0 & 0 & 0 & 1 & 1 & 0 & 1 & 1 & 0 & 1 & 0 & 0 & 0 & 1 & 0 & 1 & 0 & 0 & 0 & 0 & 0 \\
\hline 0 & 0 & 0 & 0 & 0 & 0 & 0 & 2 & 3 & 2 & 0 & 1 & 1 & 0 & 0 & 1 & 3 & 2 & 1 & 2 & 0 & 0 & 0 & 0 \\
\hline 0 & 0 & 0 & 0 & 0 & 1 & 1 & 2 & 2 & 1 & 0 & 1 & 0 & 1 & 2 & 1 & 1 & 1 & 2 & 0 & 1 & 0 & 1 & 0 \\
\hline 0 & 0 & 0 & 0 & 0 & 0 & 1 & 2 & 1 & 1 & 1 & 0 & 0 & 0 & 0 & 2 & 1 & 1 & 3 & 0 & 0 & 1 & 1 & 0 \\
\hline 0 & 0 & 0 & 0 & 0 & 0 & 2 & 1 & 2 & 3 & 1 & 0 & 1 & 0 & 0 & 1 & 0 & 2 & 0 & 1 & 1 & 0 & 1 & 0 \\
\hline 0 & 0 & 0 & 0 & 0 & 0 & 1 & 0 & 0 & 0 & 1 & 1 & 1 & 2 & 1 & 0 & 0 & 0 & 0 & 0 & 1 & 0 & 0 & 1 \\
\hline 0 & 0 & 0 & 0 & 0 & 0 & 0 & 3 & 0 & 0 & 1 & 0 & 0 & 0 & 1 & 0 & 2 & 0 & 1 & 0 & 0 & 0 & 0 & 0 \\
\hline 0 & 0 & 0 & 0 & 0 & 1 & 2 & 1 & 1 & 1 & 1 & 0 & 2 & 0 & 1 & 4 & 0 & 1 & 1 & 1 & 1 & 0 & 0 & 0 \\
\hline 0 & 0 & 0 & 0 & 0 & 0 & 1 & 1 & 3 & 2 & 0 & 1 & 2 & 0 & 2 & 0 & 1 & 2 & 1 & 0 & 1 & 1 & 0 & 0 \\
\hline 0 & 0 & 0 & 0 & 0 & 0 & 1 & 1 & 1 & 1 & 0 & 1 & 1 & 0 & 1 & 1 & 2 & 2 & 1 & 0 & 0 & 0 & 0 & 0 \\
\hline 0 & 0 & 0 & 0 & 0 & 0 & 1 & 3 & 1 & 0 & 2 & 1 & 1 & 0 & 1 & 1 & 1 & 1 & 0 & 1 & 0 & 0 & 0 & 0 \\
\hline 0 & 0 & 0 & 0 & 0 & 0 & 0 & 0 & 0 & 0 & 0 & 0 & 0 & 0 & 0 & 0 & 0 & 0 & 0 & 0 & 0 & 1 & 0 & 0 \\
\hline 0 & 0 & 0 & 0 & 0 & 0 & 1 & 0 & 0 & 0 & 0 & 0 & 0 & 0 & 0 & 0 & 0 & 0 & 0 & 0 & 0 & 0 & 0 & 0 \\
\hline 0 & 0 & 0 & 0 & 0 & 0 & 0 & 1 & 1 & 0 & 0 & 0 & 1 & 0 & 0 & 1 & 1 & 1 & 0 & 0 & 0 & 0 & 0 & 0 \\
\hline 0 & 0 & 0 & 0 & 0 & 1 & 1 & 0 & 1 & 0 & 1 & 1 & 0 & 0 & 0 & 0 & 2 & 0 & 0 & 0 & 0 & 0 & 0 & 0 \\
\hline 0 & 0 & 0 & 0 & 0 & 0 & 1 & 0 & 0 & 1 & 1 & 1 & 0 & 1 & 1 & 0 & 1 & 1 & 0 & 1 & 0 & 0 & 0 & 0 \\
\hline 0 & 0 & 0 & 0 & 0 & 0 & 1 & 3 & 1 & 1 & 1 & 0 & 0 & 0 & 0 & 1 & 0 & 1 & 0 & 1 & 0 & 0 & 0 & 1 \\
\hline
\end{tabular}

Table 10: Train distribution of the non-cyclic timetable under the 2014 demand 


\begin{tabular}{|c|c|c|c|c|c|c|c|c|c|c|c|c|c|c|c|c|c|c|c|c|c|c|c|}
\hline 0 & 1 & 2 & 3 & 4 & 5 & 6 & 7 & 8 & 9 & 10 & 11 & 12 & 13 & 14 & 15 & 16 & 17 & 18 & 19 & 20 & 21 & 22 & 23 \\
\hline 0 & 0 & 0 & 0 & 0 & 1 & 2 & 1 & 1 & 1 & 1 & 1 & 1 & 1 & 0 & 2 & 1 & 1 & 1 & 0 & 1 & 1 & 0 & 1 \\
\hline 0 & 0 & 0 & 0 & 0 & 0 & 1 & 1 & 1 & 1 & 1 & 1 & 1 & 1 & 1 & 1 & 1 & 1 & 1 & 2 & 0 & 1 & 2 & 0 \\
\hline 0 & 0 & 0 & 0 & 0 & 0 & 1 & 0 & 0 & 0 & 0 & 0 & 0 & 0 & 0 & 0 & 0 & 0 & 0 & 0 & 0 & 0 & 0 & 0 \\
\hline 0 & 0 & 0 & 0 & 0 & 0 & 0 & 0 & 0 & 0 & 0 & 0 & 0 & 0 & 0 & 0 & 0 & 1 & 0 & 0 & 0 & 0 & 0 & 0 \\
\hline 0 & 0 & 0 & 0 & 1 & 1 & 1 & 1 & 1 & 2 & 0 & 2 & 0 & 1 & 1 & 1 & 1 & 1 & 1 & 0 & 1 & 0 & 0 & 0 \\
\hline 0 & 0 & 0 & 0 & 0 & 0 & 1 & 2 & 1 & 1 & 1 & 0 & 1 & 1 & 1 & 1 & 2 & 1 & 1 & 1 & 1 & 0 & 1 & 0 \\
\hline 0 & 0 & 0 & 0 & 0 & 0 & 1 & 1 & 2 & 0 & 0 & 1 & 0 & 0 & 1 & 1 & 1 & 2 & 0 & 1 & 0 & 0 & 0 & 0 \\
\hline 0 & 0 & 0 & 0 & 0 & 0 & 2 & 1 & 1 & 1 & 1 & 1 & 0 & 0 & 0 & 1 & 1 & 0 & 2 & 0 & 0 & 0 & 0 & 0 \\
\hline 0 & 0 & 0 & 0 & 0 & 0 & 1 & 1 & 1 & 1 & 1 & 0 & 0 & 0 & 1 & 1 & 1 & 1 & 1 & 1 & 1 & 1 & 0 & 0 \\
\hline 0 & 0 & 0 & 0 & 0 & 0 & 1 & 2 & 1 & 1 & 1 & 1 & 1 & 0 & 1 & 1 & 1 & 1 & 0 & 0 & 0 & 0 & 0 & 0 \\
\hline 0 & 0 & 0 & 0 & 0 & 0 & 1 & 2 & 1 & 1 & 1 & 0 & 1 & 1 & 0 & 2 & 0 & 2 & 0 & 1 & 1 & 1 & 0 & 0 \\
\hline 0 & 0 & 0 & 0 & 0 & 0 & 1 & 1 & 1 & 1 & 0 & 1 & 0 & 1 & 0 & 2 & 1 & 1 & 1 & 1 & 1 & 1 & 0 & 1 \\
\hline 0 & 0 & 0 & 0 & 0 & 1 & 1 & 2 & 1 & 1 & 0 & 1 & 1 & 1 & 1 & 2 & 1 & 1 & 1 & 1 & 1 & 1 & 0 & 0 \\
\hline 0 & 0 & 0 & 0 & 1 & 1 & 2 & 1 & 1 & 1 & 1 & 1 & 1 & 1 & 1 & 1 & 1 & 1 & 1 & 1 & 0 & 0 & 0 & 0 \\
\hline 0 & 0 & 0 & 0 & 0 & 0 & 0 & 1 & 1 & 0 & 1 & 0 & 0 & 0 & 0 & 0 & 0 & 1 & 0 & 0 & 0 & 0 & 0 & 0 \\
\hline 0 & 0 & 0 & 0 & 0 & 0 & 0 & 0 & 1 & 0 & 0 & 0 & 0 & 0 & 0 & 1 & 0 & 1 & 0 & 1 & 0 & 0 & 0 & 0 \\
\hline 0 & 0 & 0 & 0 & 0 & 0 & 1 & 1 & 0 & 1 & 1 & 0 & 0 & 0 & 0 & 1 & 1 & 0 & 1 & 0 & 0 & 0 & 0 & 0 \\
\hline 0 & 0 & 0 & 0 & 0 & 0 & 0 & 1 & 0 & 1 & 0 & 1 & 0 & 0 & 0 & 1 & 1 & 1 & 0 & 1 & 0 & 0 & 0 & 0 \\
\hline 0 & 0 & 0 & 0 & 0 & 0 & 2 & 1 & 1 & 1 & 1 & 1 & 1 & 0 & 1 & 0 & 2 & 1 & 1 & 1 & 1 & 1 & 1 & 1 \\
\hline 0 & 0 & 0 & 0 & 0 & 0 & 1 & 1 & 1 & 1 & 1 & 1 & 1 & 1 & 1 & 1 & 0 & 1 & 1 & 1 & 1 & 1 & 1 & 1 \\
\hline 0 & 0 & 0 & 0 & 0 & 0 & 0 & 1 & 2 & 1 & 1 & 0 & 1 & 1 & 1 & 0 & 2 & 0 & 1 & 1 & 2 & 0 & 0 & 1 \\
\hline 0 & 0 & 0 & 0 & 0 & 0 & 1 & 2 & 1 & 1 & 1 & 1 & 1 & 0 & 1 & 1 & 1 & 1 & 1 & 1 & 1 & 0 & 1 & 0 \\
\hline 0 & 0 & 0 & 0 & 0 & 0 & 1 & 0 & 0 & 1 & 0 & 1 & 1 & 0 & 0 & 1 & 0 & 2 & 1 & 1 & 0 & 0 & 0 & 0 \\
\hline 0 & 0 & 0 & 0 & 0 & 1 & 1 & 2 & 1 & 0 & 0 & 0 & 0 & 0 & 0 & 0 & 2 & 1 & 0 & 0 & 0 & 0 & 0 & 0 \\
\hline 0 & 0 & 0 & 0 & 0 & 1 & 1 & 1 & 1 & 1 & 1 & 1 & 1 & 1 & 1 & 1 & 1 & 1 & 1 & 1 & 1 & 1 & 1 & 0 \\
\hline 0 & 0 & 0 & 0 & 0 & 1 & 1 & 2 & 0 & 2 & 1 & 1 & 0 & 2 & 1 & 1 & 1 & 1 & 1 & 1 & 1 & 1 & 0 & 0 \\
\hline 0 & 0 & 0 & 0 & 0 & 0 & 1 & 2 & 1 & 0 & 1 & 1 & 1 & 2 & 0 & 2 & 1 & 1 & 0 & 0 & 0 & 0 & 0 & 0 \\
\hline 0 & 0 & 0 & 0 & 0 & 0 & 2 & 1 & 0 & 2 & 1 & 1 & 1 & 1 & 1 & 1 & 0 & 1 & 1 & 1 & 0 & 0 & 0 & 0 \\
\hline 0 & 0 & 0 & 0 & 0 & 0 & 0 & 0 & 0 & 0 & 0 & 0 & 0 & 0 & 0 & 0 & 0 & 1 & 0 & 0 & 0 & 0 & 0 & 0 \\
\hline 0 & 0 & 0 & 0 & 0 & 0 & 0 & 0 & 0 & 0 & 0 & 0 & 0 & 0 & 0 & 0 & 0 & 1 & 0 & 0 & 0 & 0 & 0 & 0 \\
\hline 0 & 0 & 0 & 0 & 0 & 0 & 0 & 0 & 0 & 1 & 0 & 1 & 0 & 1 & 0 & 0 & 2 & 1 & 0 & 0 & 0 & 0 & 0 & 0 \\
\hline 0 & 0 & 0 & 0 & 0 & 0 & 1 & 1 & 1 & 0 & 0 & 1 & 0 & 1 & 0 & 1 & 0 & 0 & 0 & 0 & 0 & 0 & 0 & 1 \\
\hline 0 & 0 & 0 & 0 & 0 & 0 & 1 & 1 & 1 & 0 & 0 & 1 & 1 & 0 & 1 & 1 & 0 & 1 & 1 & 0 & 0 & 0 & 0 & 0 \\
\hline 0 & 0 & 0 & 0 & 0 & 0 & 1 & 2 & 0 & 2 & 0 & 1 & 1 & 0 & 0 & 0 & 1 & 1 & 0 & 0 & 0 & 1 & 1 & 0 \\
\hline
\end{tabular}

Table 11: Train distribution of the 30 min shifted cyclic timetable under the 2014 demand 


\begin{tabular}{|c|c|c|c|c|c|c|c|c|c|c|c|c|c|c|c|c|c|c|c|c|c|c|c|}
\hline 0 & 1 & 2 & 3 & 4 & 5 & 6 & 7 & 8 & 9 & 10 & 11 & 12 & 13 & 14 & 15 & 16 & 17 & 18 & 19 & 20 & 21 & 22 & 23 \\
\hline 0 & 0 & 0 & 0 & 0 & 1 & 2 & 2 & 1 & 2 & 1 & 1 & 0 & 1 & 1 & 1 & 1 & 0 & 2 & 0 & 1 & 1 & 0 & 0 \\
\hline 0 & 0 & 0 & 0 & 0 & 0 & 1 & 1 & 1 & 1 & 1 & 1 & 1 & 1 & 1 & 1 & 2 & 1 & 1 & 1 & 1 & 1 & 0 & 1 \\
\hline 0 & 0 & 0 & 0 & 0 & 0 & 1 & 0 & 0 & 0 & 0 & 0 & 0 & 0 & 0 & 0 & 0 & 0 & 0 & 0 & 0 & 0 & 0 & 0 \\
\hline 0 & 0 & 0 & 0 & 0 & 0 & 0 & 0 & 0 & 0 & 0 & 0 & 0 & 0 & 0 & 0 & 0 & 0 & 0 & 0 & 0 & 0 & 0 & 0 \\
\hline 0 & 0 & 0 & 0 & 0 & 0 & 3 & 2 & 2 & 1 & 0 & 0 & 2 & 0 & 1 & 1 & 2 & 1 & 0 & 0 & 0 & 1 & 0 & 0 \\
\hline 0 & 0 & 0 & 0 & 0 & 0 & 1 & 1 & 2 & 0 & 1 & 1 & 0 & 1 & 1 & 3 & 1 & 2 & 0 & 1 & 1 & 0 & 1 & 0 \\
\hline 0 & 0 & 0 & 0 & 0 & 0 & 1 & 0 & 1 & 1 & 0 & 1 & 1 & 0 & 1 & 1 & 1 & 1 & 0 & 1 & 1 & 0 & 0 & 0 \\
\hline 0 & 0 & 0 & 0 & 0 & 0 & 1 & 1 & 3 & 2 & 0 & 0 & 0 & 0 & 0 & 0 & 1 & 1 & 2 & 0 & 0 & 0 & 0 & 0 \\
\hline 0 & 0 & 0 & 0 & 0 & 0 & 1 & 3 & 0 & 2 & 0 & 0 & 0 & 0 & 0 & 2 & 2 & 1 & 0 & 2 & 0 & 0 & 0 & 0 \\
\hline 0 & 0 & 0 & 0 & 0 & 0 & 2 & 1 & 3 & 0 & 2 & 0 & 0 & 0 & 2 & 0 & 1 & 1 & 0 & 0 & 0 & 0 & 0 & 0 \\
\hline 0 & 0 & 0 & 0 & 0 & 0 & 2 & 2 & 2 & 1 & 1 & 1 & 0 & 0 & 1 & 1 & 1 & 1 & 1 & 1 & 0 & 0 & 0 & 0 \\
\hline 0 & 0 & 0 & 0 & 0 & 0 & 0 & 2 & 1 & 0 & 0 & 0 & 2 & 1 & 2 & 1 & 2 & 1 & 2 & 1 & 0 & 0 & 1 & 0 \\
\hline 0 & 0 & 0 & 0 & 0 & 1 & 1 & 3 & 1 & 1 & 0 & 2 & 1 & 1 & 1 & 2 & 1 & 3 & 0 & 0 & 0 & 0 & 0 & 0 \\
\hline 0 & 0 & 0 & 0 & 0 & 1 & 1 & 1 & 2 & 1 & 1 & 1 & 1 & 1 & 1 & 1 & 2 & 1 & 1 & 1 & 0 & 0 & 0 & 0 \\
\hline 0 & 0 & 0 & 0 & 0 & 0 & 0 & 1 & 0 & 0 & 0 & 0 & 0 & 0 & 0 & 0 & 1 & 0 & 0 & 0 & 0 & 0 & 0 & 0 \\
\hline 0 & 0 & 0 & 0 & 0 & 0 & 0 & 0 & 1 & 0 & 0 & 0 & 0 & 0 & 0 & 0 & 0 & 0 & 1 & 0 & 0 & 0 & 0 & 0 \\
\hline 0 & 0 & 0 & 0 & 0 & 0 & 1 & 1 & 0 & 1 & 0 & 0 & 0 & 0 & 0 & 1 & 0 & 0 & 2 & 1 & 0 & 0 & 0 & 0 \\
\hline 0 & 0 & 0 & 0 & 0 & 0 & 1 & 2 & 0 & 0 & 1 & 1 & 0 & 0 & 0 & 0 & 1 & 0 & 0 & 0 & 1 & 0 & 0 & 0 \\
\hline 0 & 0 & 0 & 0 & 0 & 0 & 2 & 1 & 1 & 1 & 0 & 1 & 0 & 1 & 2 & 1 & 3 & 1 & 1 & 1 & 1 & 0 & 1 & 0 \\
\hline 0 & 0 & 0 & 0 & 0 & 0 & 2 & 3 & 1 & 1 & 1 & 0 & 2 & 0 & 0 & 1 & 1 & 1 & 2 & 2 & 0 & 1 & 0 & 0 \\
\hline 0 & 0 & 0 & 0 & 0 & 0 & 1 & 1 & 2 & 0 & 0 & 1 & 3 & 1 & 0 & 3 & 1 & 1 & 1 & 0 & 0 & 0 & 0 & 0 \\
\hline 0 & 0 & 0 & 0 & 0 & 1 & 2 & 1 & 1 & 1 & 1 & 1 & 1 & 0 & 1 & 1 & 1 & 1 & 2 & 0 & 1 & 0 & 0 & 0 \\
\hline 0 & 0 & 0 & 0 & 0 & 0 & 0 & 0 & 1 & 2 & 1 & 1 & 0 & 0 & 0 & 0 & 0 & 1 & 1 & 1 & 0 & 1 & 0 & 0 \\
\hline 0 & 0 & 0 & 0 & 0 & 0 & 0 & 1 & 3 & 0 & 0 & 0 & 0 & 2 & 0 & 0 & 0 & 0 & 0 & 0 & 0 & 0 & 1 & 0 \\
\hline 0 & 0 & 0 & 0 & 0 & 1 & 1 & 1 & 1 & 0 & 1 & 1 & 0 & 1 & 2 & 2 & 1 & 2 & 2 & 1 & 1 & 0 & 0 & 0 \\
\hline 0 & 0 & 0 & 0 & 0 & 1 & 3 & 1 & 2 & 2 & 1 & 0 & 0 & 1 & 0 & 2 & 1 & 1 & 0 & 1 & 1 & 1 & 0 & 0 \\
\hline 0 & 0 & 0 & 0 & 0 & 0 & 1 & 0 & 2 & 2 & 0 & 1 & 0 & 1 & 1 & 1 & 2 & 0 & 0 & 2 & 0 & 0 & 0 & 0 \\
\hline 0 & 0 & 0 & 0 & 0 & 0 & 0 & 3 & 1 & 1 & 2 & 0 & 2 & 0 & 1 & 1 & 1 & 0 & 2 & 0 & 0 & 0 & 0 & 0 \\
\hline 0 & 0 & 0 & 0 & 0 & 0 & 0 & 0 & 0 & 0 & 0 & 0 & 0 & 0 & 0 & 0 & 0 & 0 & 0 & 0 & 0 & 0 & 1 & 0 \\
\hline 0 & 0 & 0 & 0 & 0 & 0 & 0 & 0 & 0 & 0 & 0 & 0 & 0 & 0 & 0 & 1 & 0 & 0 & 0 & 0 & 0 & 0 & 0 & 0 \\
\hline 0 & 0 & 0 & 0 & 0 & 0 & 1 & 0 & 0 & 0 & 0 & 0 & 0 & 1 & 0 & 0 & 1 & 0 & 3 & 0 & 0 & 0 & 0 & 0 \\
\hline 0 & 0 & 0 & 0 & 0 & 0 & 0 & 2 & 1 & 1 & 1 & 0 & 2 & 0 & 0 & 0 & 0 & 0 & 0 & 0 & 0 & 0 & 0 & 0 \\
\hline 0 & 0 & 0 & 0 & 0 & 0 & 0 & 0 & 1 & 0 & 0 & 1 & 0 & 1 & 1 & 1 & 1 & 1 & 0 & 0 & 0 & 1 & 1 & 0 \\
\hline 0 & 0 & 0 & 0 & 0 & 0 & 2 & 2 & 0 & 0 & 0 & 1 & 0 & 1 & 1 & 1 & 0 & 0 & 1 & 0 & 0 & 0 & 0 & 1 \\
\hline
\end{tabular}

Table 12: Train distribution of the hybrid cyclic timetable under the 2014 demand 